Supporting Information for

\title{
Characterization of a sesquiterpene synthase catalyzing formation of cedrol and two diastereoisomers of tricho- acorenol from Euphorbia fischeriana
}

Jianxun Zhu, ${ }^{\dagger} \wedge$ Lihong Liu,,${ }^{\dagger} \wedge$ Maobo Wu,${ }^{\dagger}$ Guiyang Xia, ${ }^{\ddagger}$ Pengcheng Lin,,$\S$ Jiachen $\mathrm{Zi}^{\dagger}, *$

$\dagger$ College of Pharmacy, Jinan University, Guangzhou 510632, People’s Republic of China

\$ Key Laboratory of Chinese Internal Medicine of Ministry of Education and Beijing, Dongzhimen Hospital, Beijing University of Chinese Medicine, Beijing 100700, People's Republic of China

$\S$ College of Pharmacy, Qinghai Nationalities University, Xining 810007, People’s Republic of China

* Corresponding Author Jiachen Zi - College of Pharmacy, Jinan University, Guangzhou 510632, China; orcid.org/0000-0002-0212-1282; Phone:+86-02-85220386; Email: tzjc@jnu.edu.cn

${ }^{\wedge}$ These authors contribute equally to this paper. 


\section{Contents}

Table S1. Terpene synthases used for performing BLAST analysis against the transcriptome database.

Figure S1. Phylogenetic analysis of EfTPS12 and the previously characterized terpene synthases from other species.

Table S2. Terpene synthases used for construction of phylogenetic tree.............5

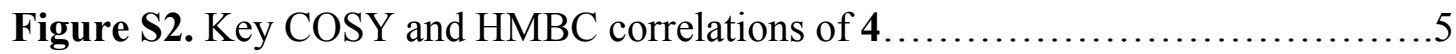

In silico prediction of ECD spectra........................................... 5

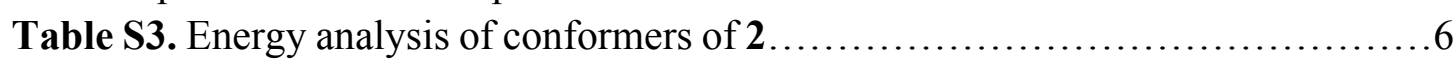

Figure S3. B3LYP/6-311+G(d,p) optimized two lowest energy 3D conformers of $2 \ldots 7$

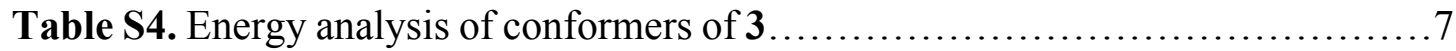

Figure S4. B3LYP/6-311+G(d,p) optimized three lowest energy 3D conformers of 3...8

Figure S5. GC-MS chromatograms of the extracts of the reaction mixtures of tris(tetrabutyl ammonium) 12-hydroxyfarnesyl diphosphate with either EfTPS12 (red) or boiled EfTPS12 (black) ..................................................... 8

Figure S6. GC-MS chromatogram of the extract of the culture of the E. coli strains coexpressing ERG20 and each EfTPS12 mutant...............................9

Figure S7. GC-MS chromatograms of the extracts of the cultures of the E. coli BL21 strains containing petDuet-1-ERG20-EfTPS12 (BL21-EfTPS12, red) or petDuet-1ERG20 (BL21-CK, black)...............................................9

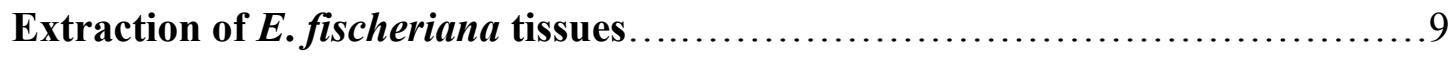

Figure S8. GC-MS chromatograms of the extracts of roots, stems and leaves of $E$.

fischeriana ........................................................... 10

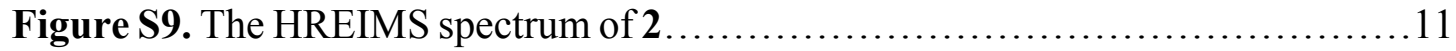

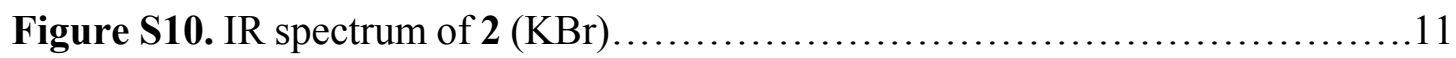

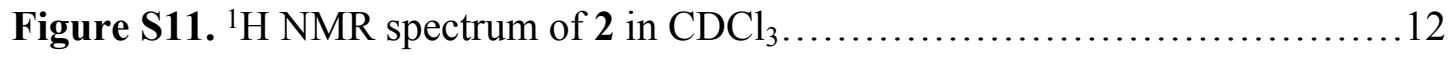

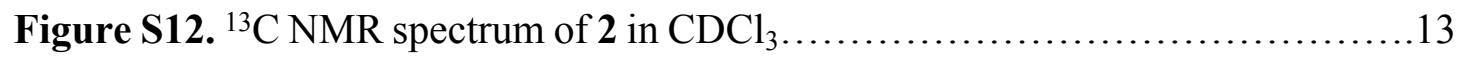

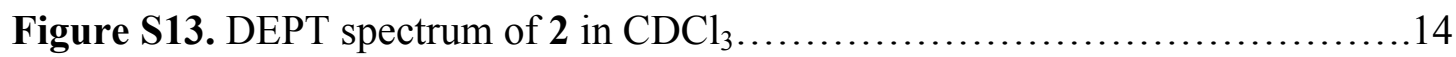

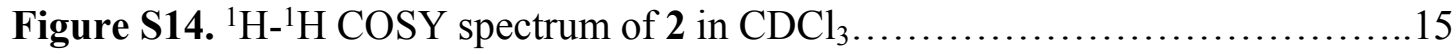

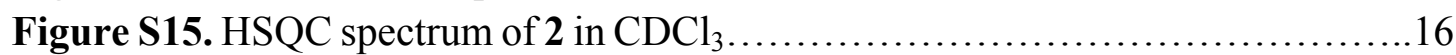

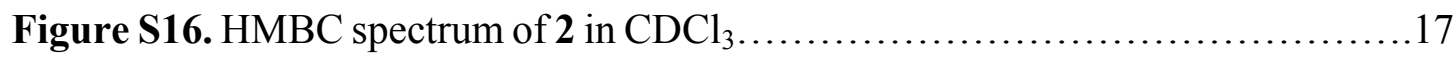

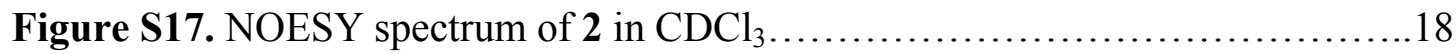

Figure S18. NOESY spectrum (lower contour level) of $\mathbf{2}$ in $\mathrm{CDCl}_{3} \ldots \ldots \ldots \ldots \ldots \ldots \ldots$

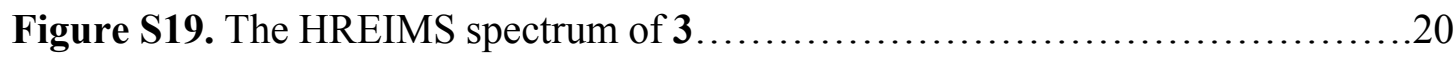

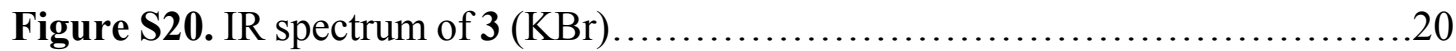

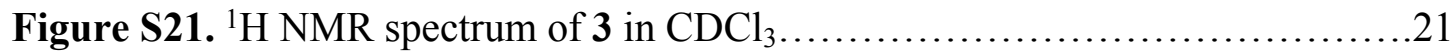

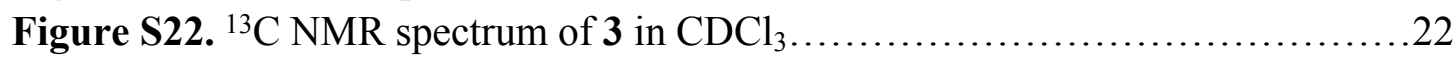

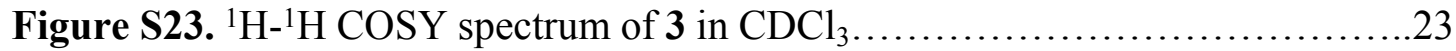

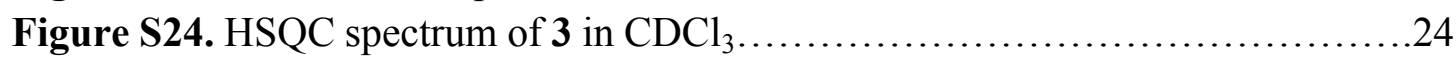

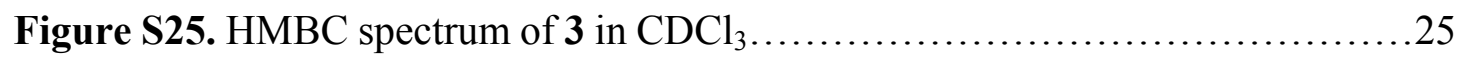

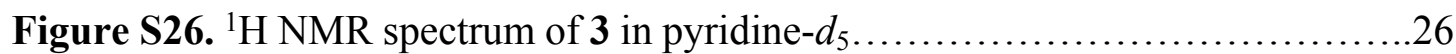




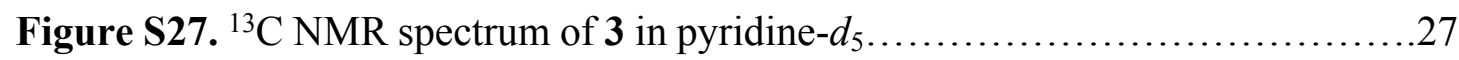

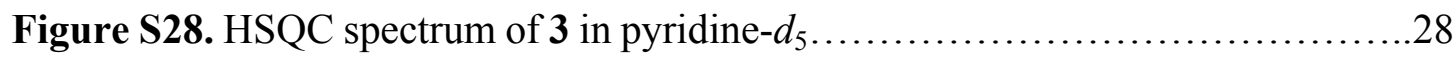

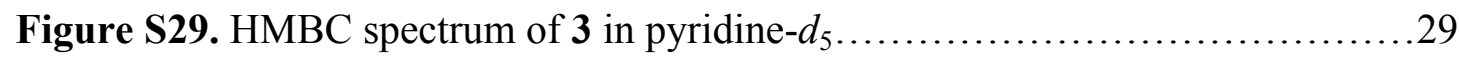

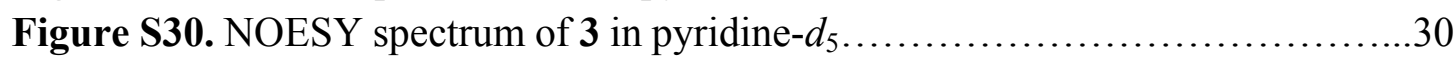

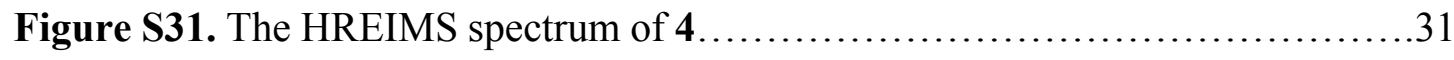

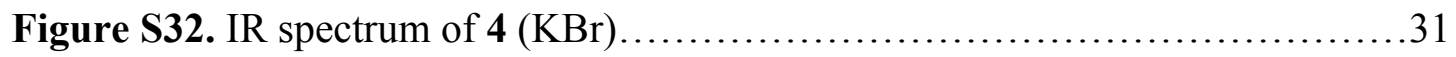

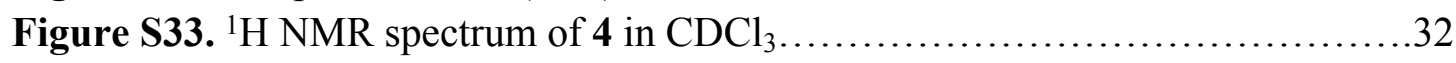

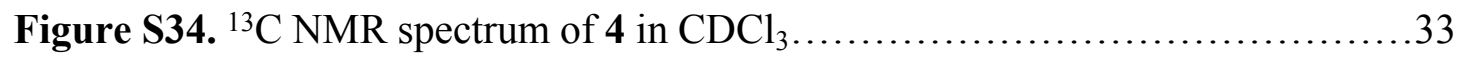

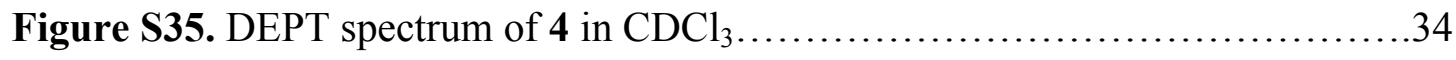

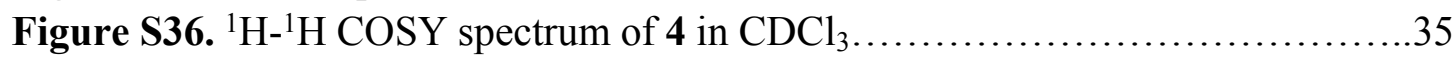

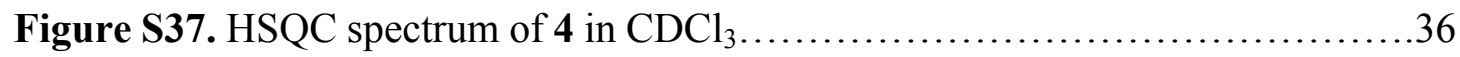

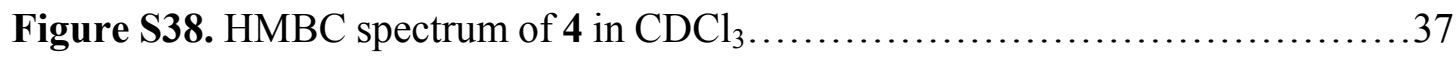

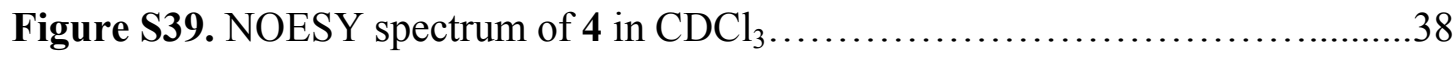

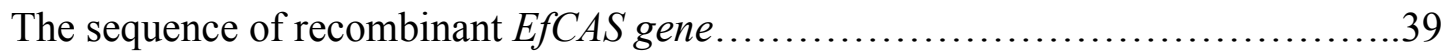

The sequence of recombinant EfCAS ....................................... 39 
Table S1. Terpene synthases used for performing BLAST analysis against the transcriptome database

\begin{tabular}{ccc}
\hline Species & Name & Accession No. \\
\hline bidopsis thaliana & $(Z)$ - $\gamma$-bisabolene synthase & NP_001328007.1 \\
Zea mays & $(S)$ - $\beta$-macrocarpene synthase & NP_001307938.1 \\
Zingiber officinale & $\beta$-bisabolene synthase & BAI67934.1 \\
Capsicum annuum & 5 -epi-aristolochene synthase & CAA06614.1 \\
Nicotiana tabacum & 5 -epi-aristolochene synthase & AAO85555.1 \\
Helianthus annuus & $\gamma$-bisabolene synthase & AME16498.1 \\
Santalum austrocaledonicum & $\beta$-bisabolene synthase & ADO87003.1 \\
Santalum spicatum & $\alpha$-bisabolol synthase & E3W206.1 \\
Santalum spicatum & santalene synthase & ADO87002.1 \\
Santalum album & santalene synthase & ADO87000.1 \\
Santalum austrocaledonicum & santalene synthase & ADO87001.1 \\
Pseudotsuga menziesii & $(E)$ - $\gamma$-bisabolene synthase & Q4QSN4.1 \\
Picea abies & $(E)$ - $\alpha$-bisabolene synthase & AAS47689.1 \\
Abies grandis & $(E)$ - $\alpha$-bisabolene synthase & AAC24192.1 \\
Abies grandis & $\delta$-selinene synthase & AAC05727.1 \\
Picea abies & longifolene synthase & AAS47695.1 \\
Abies grandis & $\gamma$-humulene synthase & AAC05728.1 \\
Pinus taeda & $\alpha$-farnesene synthase & AAO61226.1 \\
\hline
\end{tabular}

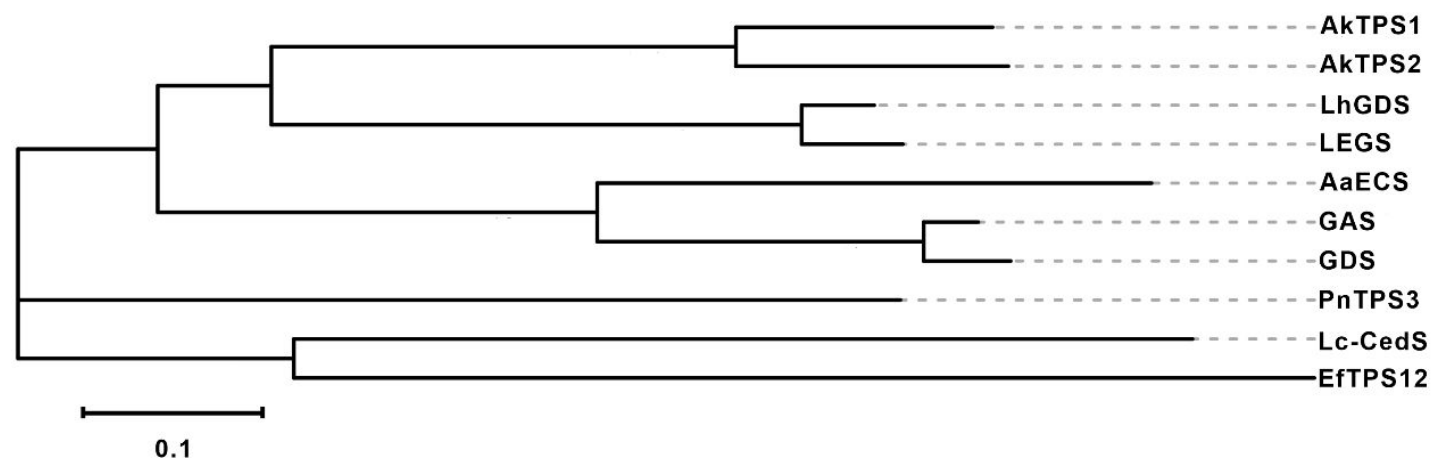

Figure S1. Phylogenetic analysis of EfTPS12 and the previously characterized terpene synthases from other species. The phylogenetic tree was created using neighbor-joining method. Accession numbers of all the terpene synthase used here were shown in Table S2. 
Table S2. Terpene synthases used for construction of phylogenetic tree

\begin{tabular}{cccc}
\hline Name & Species & Function & Accession No. \\
\hline AkTPS1 & Angelica keiskei & germacrene D synthase & BBO53954.1 \\
AkTPS2 & Angelica keiskei & germacrenes B and D synthase & BBO53955.1 \\
PnTPS3 & Piper nigrum & copaene synthase & AVY53326.1 \\
GAS & Solidago canadensis & germacrene A synthase & CAC36896.1 \\
GDS & Solidago canadensis & germacrene D synthase & CAE47440.1 \\
LhGDS & Solanum habrochaites & germacrene D synthase & AAG41892.1 \\
LEGS & Solanum lycopersicum & epidermal germacrene C synthase & AAC39431.1 \\
AaECS & Artemisia annua & epi-cedrol synthase & PWA94461.1 \\
Lc-CedS & Leucosceptrum canum & cedrol synthase & QBP05430.1 \\
\hline
\end{tabular}

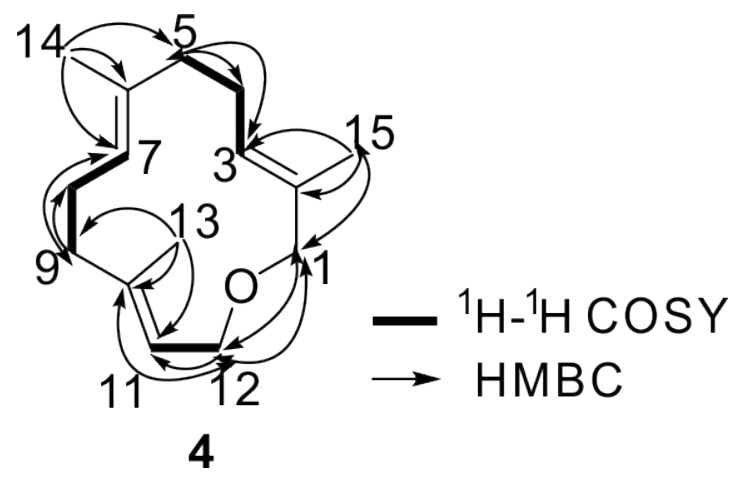

Figure S2. Key COSY and HMBC correlations of 4.

\section{In silico prediction of ECD spectra.}

All calculations were performed using Gaussian $16 .{ }^{1}$ Conformation search using molecular mechanics calculations was performed in DS (Discovery Studio) 2018 with MMFF94s force field with $20 \mathrm{kcal} \mathrm{mol}^{-1}$ upper energy limit at best level. The conformers performed with the DS 2018 software package were further optimized by using the TDDFT method at the B3LYP/6-31+G $(\mathrm{d}, \mathrm{p})$ level, and the frequency was calculated at the same level of theory. For all optimized structures, vibrational spectra were calculated to ensure that no imaginary frequencies for energy minimum were obtained. The average values were obtained by the Boltzmann distributions, using the relative Gibbs free energies as weighting factors. The stable conformers were subjected to ECD calculation by the TDDFT method at the B3LYP/6-311+G(d,p) level with the 
CPCM model in $\mathrm{MeOH}$. ECD spectra of different conformers were simulated using SpecDis $1.71^{2}$ with a half-bandwidth of $0.2 \mathrm{eV}$, and the final calculated ECD spectra were obtained according to the Boltzmann-calculated contribution of each con-former. The calculated ECD spectra were compared with the experimental data.

1. Gaussian 16, Revision A.03, M. J. Frisch, G. W. Trucks, H. B. Schlegel, G. E. Scuseria, M. A. Robb, J. R. Cheeseman, G. Scalmani, V. Barone, G. A. Petersson, H. Nakatsuji, X. Li, M. Caricato, A. V. Marenich, J. Bloino, B. G. Janesko, R. Gomperts, B. Mennucci, H. P. Hratchian, J. V. Ortiz, A. F. Izmaylov, J. L. Sonnenberg, D. Williams-Young, F. Ding, F. Lipparini, F. Egidi, J. Goings, B. Peng, A. Petrone, T. Henderson, D. Ranasinghe, V. G. Zakrzewski, J. Gao, N. Rega, G. Zheng, W. Liang, M. Hada, M. Ehara, K. Toyota, R. Fukuda, J. Hasegawa, M. Ishida, T. Nakajima, Y. Honda, O. Kitao, H. Nakai, T. Vreven, K. Throssell, J. A. Montgomery, Jr., J. E. Peralta, F. Ogliaro, M. J. Bearpark, J. J. Heyd, E. N. Brothers, K. N. Kudin, V. N. Staroverov, T. A. Keith, R. Kobayashi, J. Normand, K. Raghavachari, A. P. Rendell, J. C. Burant, S. S. Iyengar, J. Tomasi, M. Cossi, J. M. Millam, M. Klene, C. Adamo, R. Cammi, J. W. Ochterski, R. L. Martin, K. Morokuma, O. Farkas, J. B. Foresman, and D. J. Fox, Gaussian, Inc., Wallingford CT, 2016.

2. T. Bruhn, A. Schaumlöffel, Y. Hemberger, G. Pescitelli, SpecDis version 1.71, Berlin, Germany, 2017, http:/specdis-software.jimdo.com.

Table S3. Energy analysis of conformers of 2

\begin{tabular}{llrrc}
\hline & \multicolumn{2}{c}{ MMFF94 } & \multicolumn{2}{c}{ B3LYP/6-311+G $(\mathrm{d}, \mathrm{p})$} \\
\cline { 2 - 5 } conf. & $\Delta \mathrm{E}(\mathrm{kcal} / \mathrm{mol})$ & $\begin{array}{r}\text { Boltzmann } \\
\text { distribution }\end{array}$ & Extracted heats & $\begin{array}{c}\text { Boltzmann-calculated } \\
\text { contribution }(\%)\end{array}$ \\
\hline $\mathbf{2 - 1}$ & 0 & 0.441694244 & -662.6413841 & 0.26 \\
$\mathbf{2 - 2}$ & 0.234377 & 0.297309874 & -662.6461400 & 40.13 \\
$\mathbf{2 - 3}$ & 0.717768 & 0.131416069 & -662.6392465 & 0.03 \\
$\mathbf{2 - 4}$ & 0.73384 & 0.127896858 & -662.6465130 & 59.59 \\
\hline
\end{tabular}




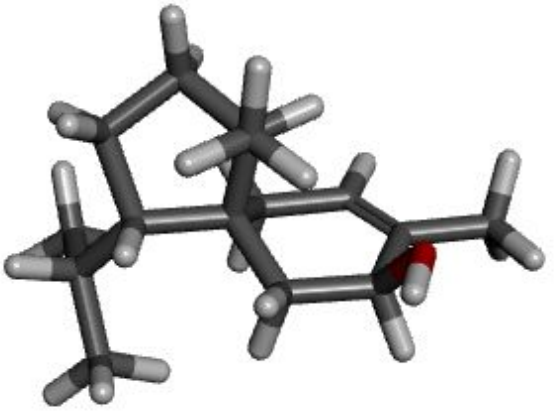

2-1

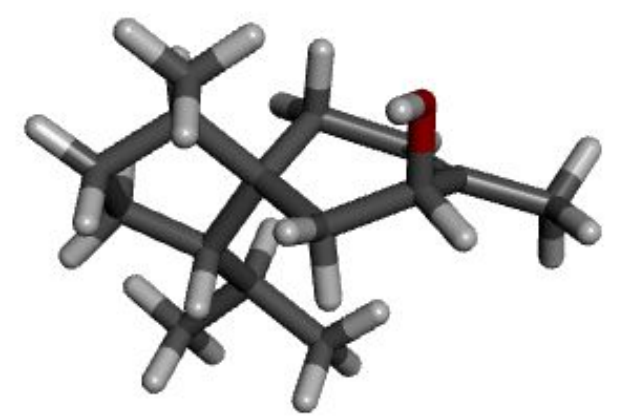

2-3

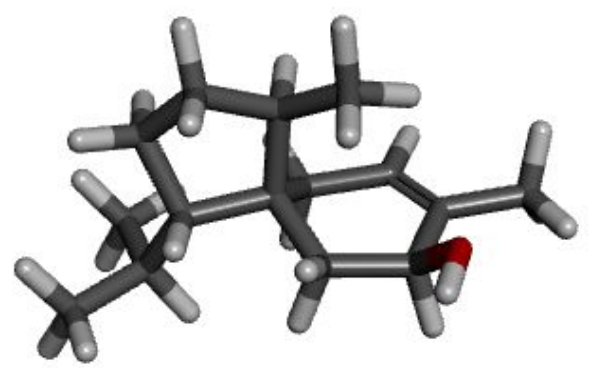

2-2

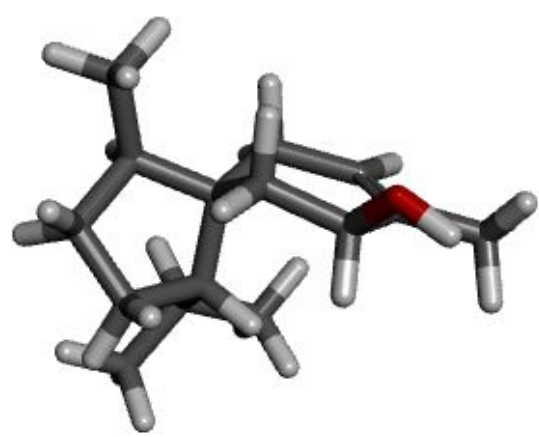

2-4

Figure S3. B3LYP/6-311+G(d,p) optimized two lowest energy 3D conformers of 2 .

Table S4. Energy analysis of conformers of 3

\begin{tabular}{ccccc}
\hline & \multicolumn{2}{c}{ MMFF94 } & \multicolumn{2}{c}{ B3LYP/6-311+G(d,p) } \\
\cline { 2 - 5 } conf. & $\Delta \mathrm{E}(\mathrm{kcal} / \mathrm{mol})$ & $\begin{array}{c}\text { Boltzmann } \\
\text { distribution }\end{array}$ & Extracted heats & $\begin{array}{c}\text { Boltzmann-calculated } \\
\text { contribution }(\%)\end{array}$ \\
\hline $\mathbf{3 - 1}$ & 0 & 0.694943029 & -662.6441024 & 1.43 \\
$\mathbf{3 - 2}$ & 0.931386 & 0.144141812 & -662.646265 & 14.20 \\
$\mathbf{3 - 3}$ & 1.30184 & 0.077102224 & -662.6475174 & 53.58 \\
$\mathbf{3 - 4}$ & 1.40776 & 0.06447272 & -662.6450594 & 3.96 \\
$\mathbf{3 - 5}$ & 2.2283 & 0.016125784 & -662.6468648 & 26.83 \\
\hline
\end{tabular}




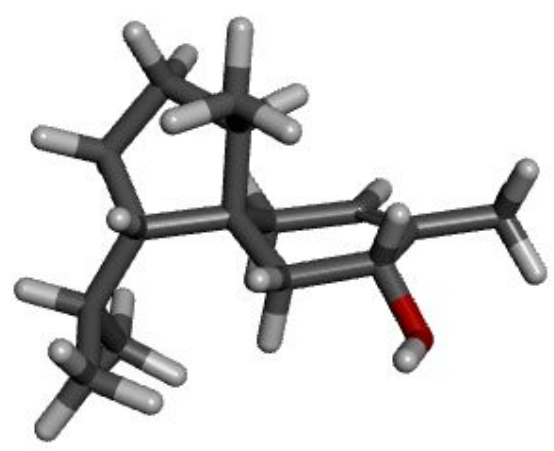

3-1

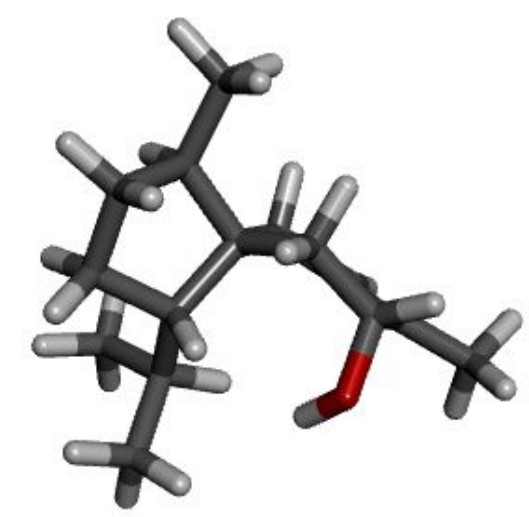

3-3

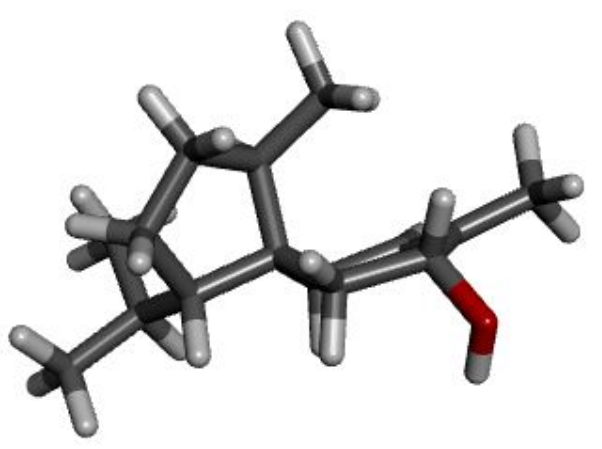

3-2

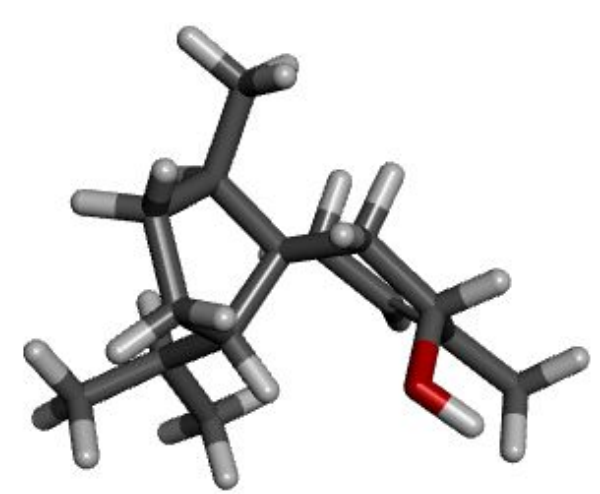

3-4

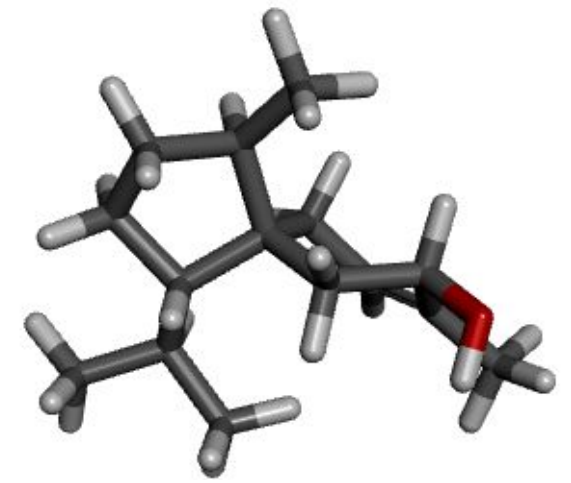

3-5

Figure S4. B3LYP/6-311+G(d,p) optimized three lowest energy 3D conformers of $\mathbf{3}$.

- Purified EfTPS12

- Boiled EfTPS12

$\begin{array}{lllllllll}13.6 & 13.8 & 14.0 & 14.2 & 14.4 & 14.6 & 14.8 & 15.0 & 15.2\end{array}$

Retention time(min)

Figure S5. GC-MS chromatograms of the extracts of the reaction mixtures of tris(tetrabutyl ammonium) 12-hydroxyfarnesyl diphosphate with either EfTPS12 (red) or boiled EfTPS12 (black). 


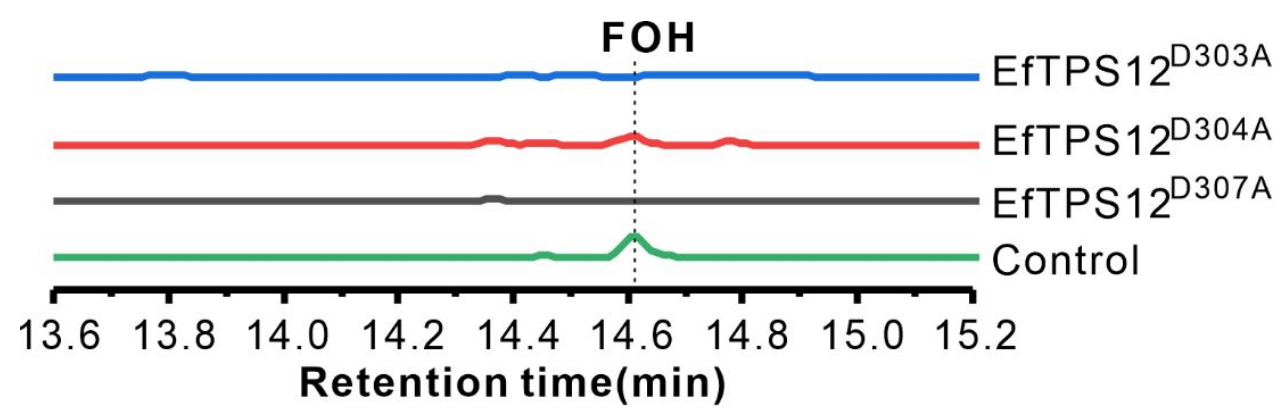

Figure S6. GC-MS chromatogram of the extract of the culture of the E. coli strain coexpressing ERG20 and each EfTPS12 mutant. Control: the E. coli strain only expressing ERG20. FOH: farnesol.

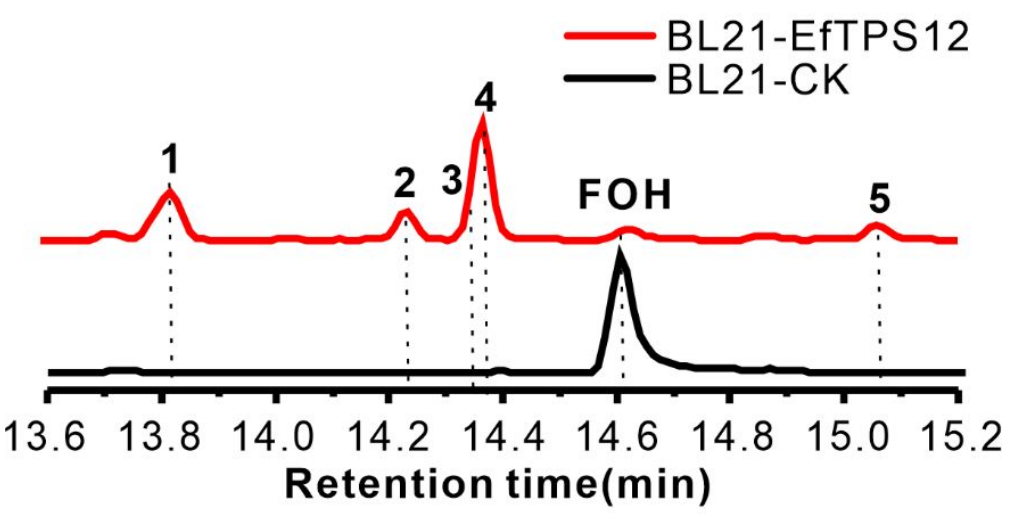

Figure S7. GC-MS chromatograms of the extracts of the cultures of the E. coli BL21 strains containing petDuet-1-ERG20-EfTPS12 (BL21-EfTPS12, red) or petDuet-1ERG20 (BL21-CK, black).

Extraction of $\boldsymbol{E}$. fischeriana tissues. Each tissue (around $10 \mathrm{~g}$ ) was frozen in liquid nitrogen and grinded to a fine powder. The material was extracted with $20 \mathrm{~mL}$ methanol. The resulting methanol solution was partitioned with $8 \mathrm{~mL}$ hexanes. The hexanes extracts were for analysis by GC-MS. 


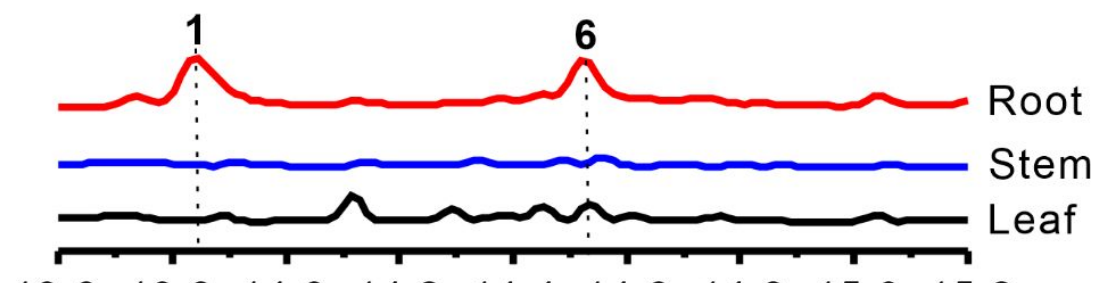

$\begin{array}{lllllllll}13.6 & 13.8 & 14.0 & 14.2 & 14.4 & 14.6 & 14.8 & 15.0 & 15.2\end{array}$

Retention time(min)

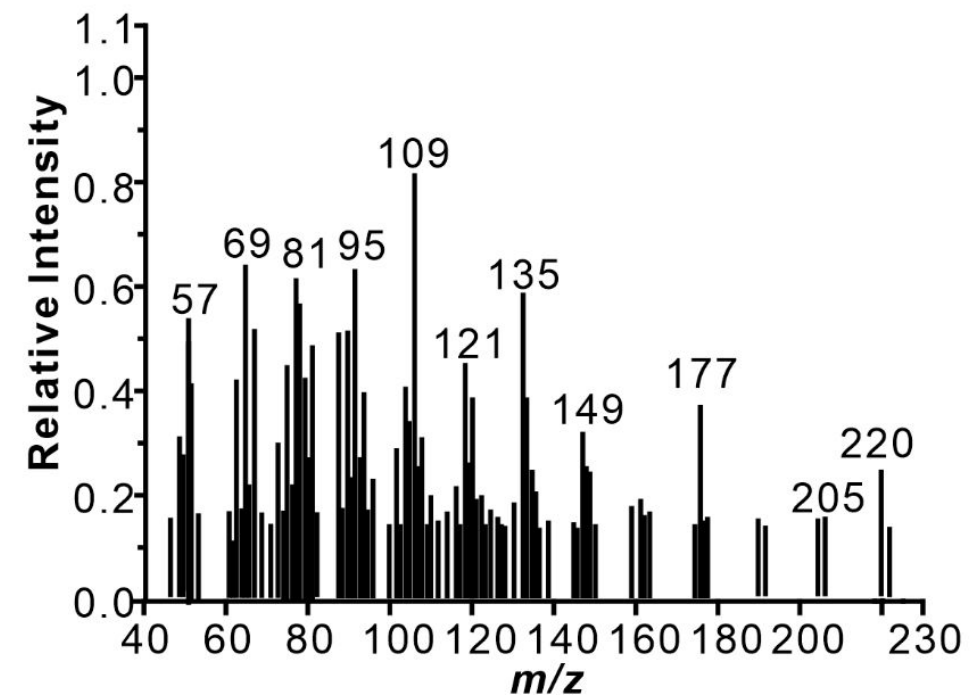

Figure S8. GC-MS chromatograms of the extracts of roots, stems and leaves of $E$. fischeriana. 1: cedrol; 6: an unknown sesquiterpene. The mass spectrum of $\mathbf{6}$ (as shown above) is very similar to that of acorenone. 


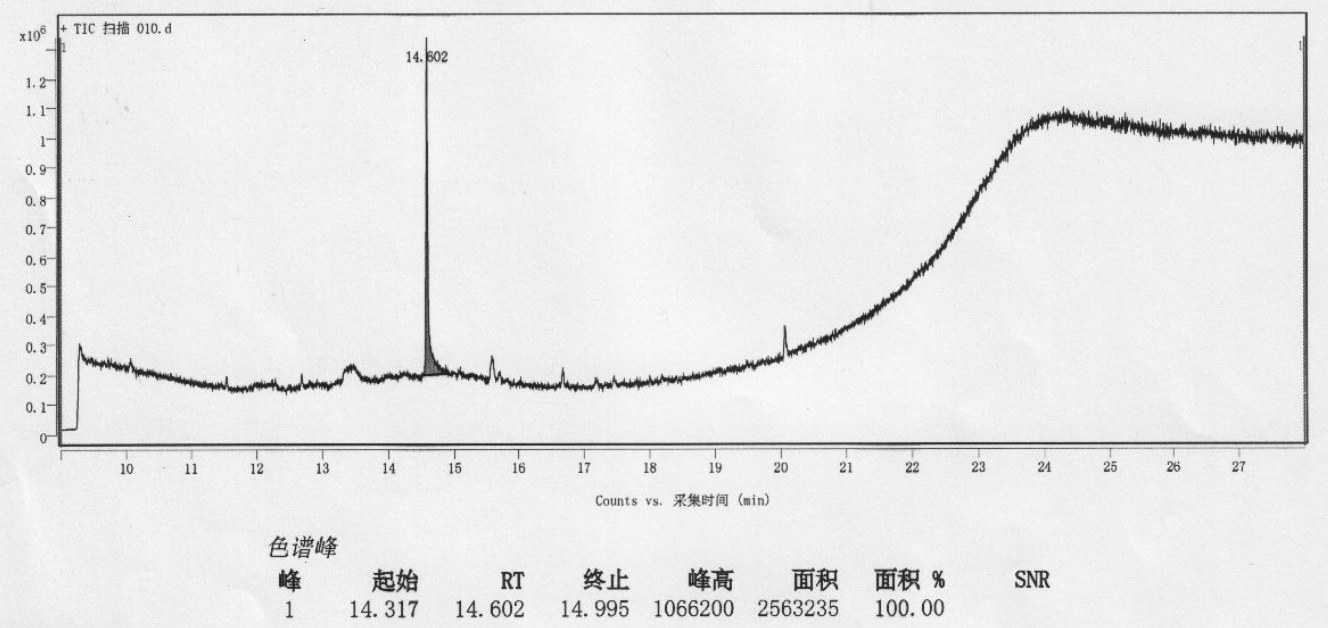

样品谱图

+ 扫描 $(r t: 14.600 \mathrm{~min})$ 扣除

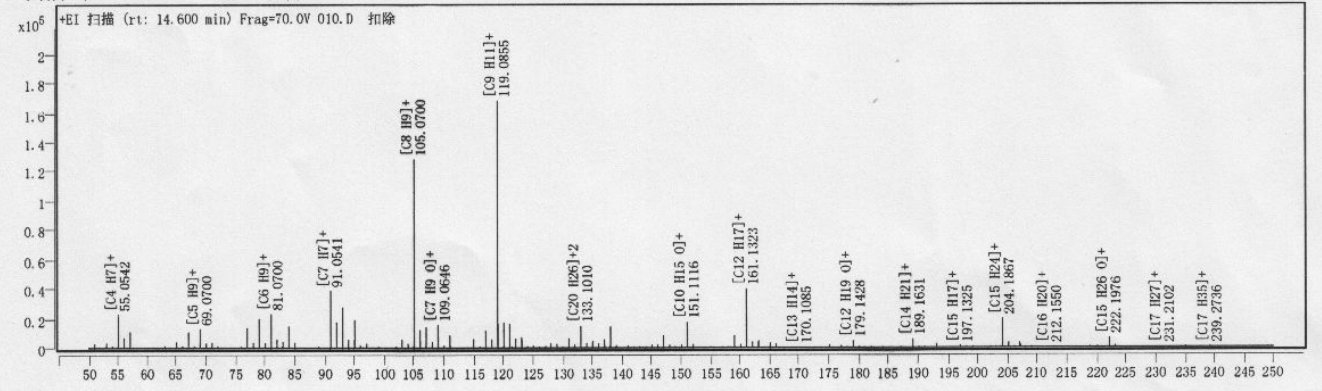

Counts vs. 原储比 $(\mathrm{m} / \mathrm{z})$

Figure S9. The HREIMS spectrum of 2.

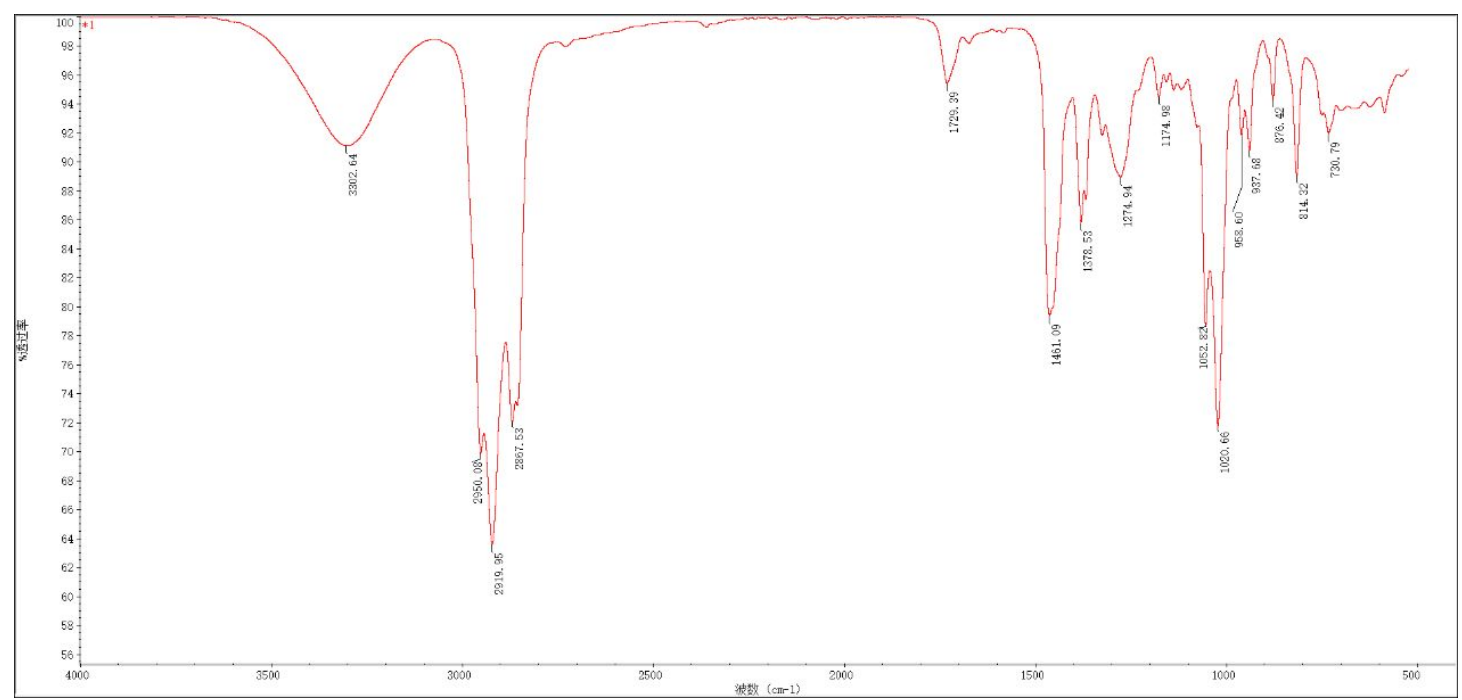

Figure S10. IR spectrum of $2(\mathrm{KBr})$ 


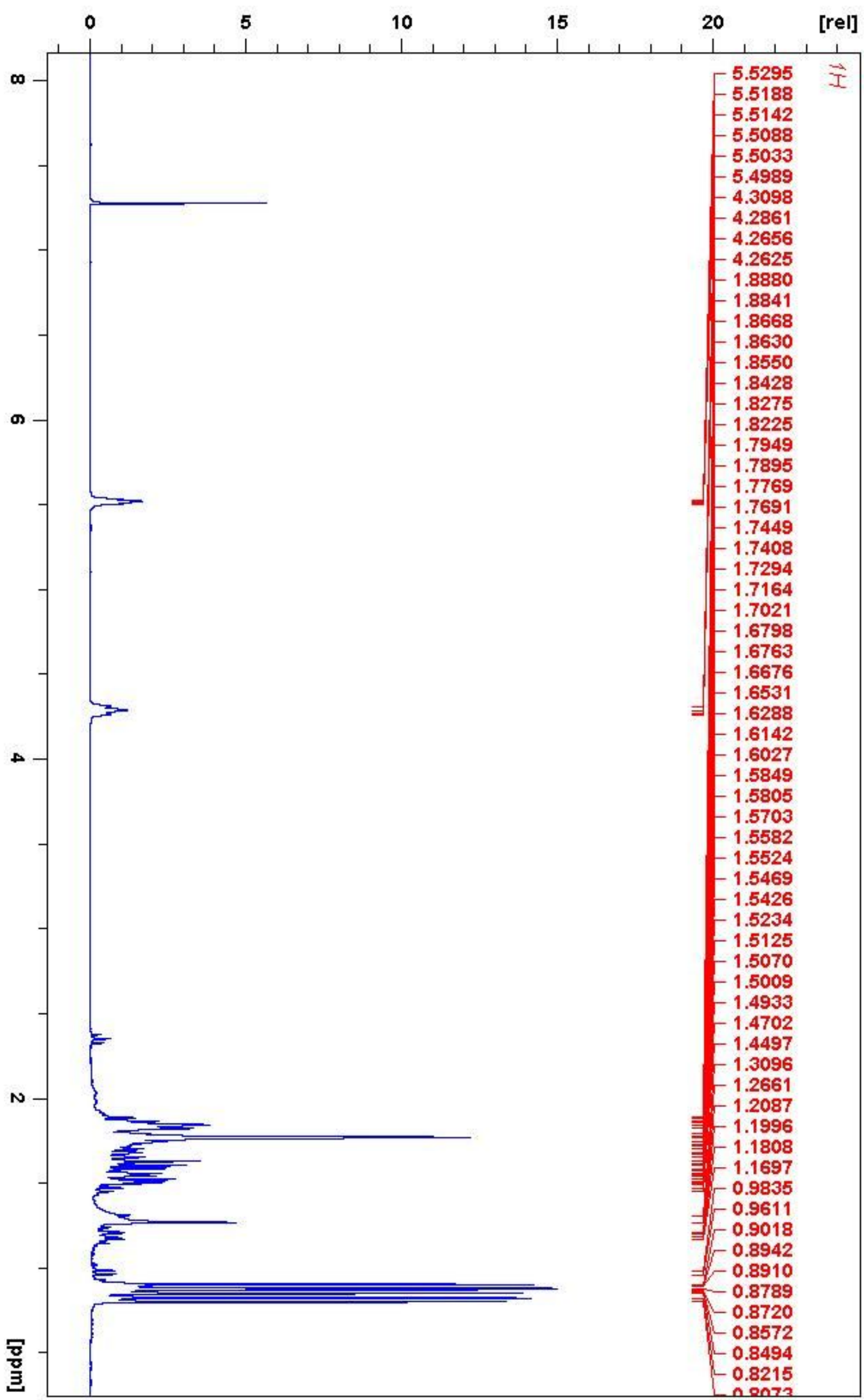

Figure S11. ${ }^{1} \mathrm{H}$ NMR spectrum of 2 in $\mathrm{CDCl}_{3}$ 


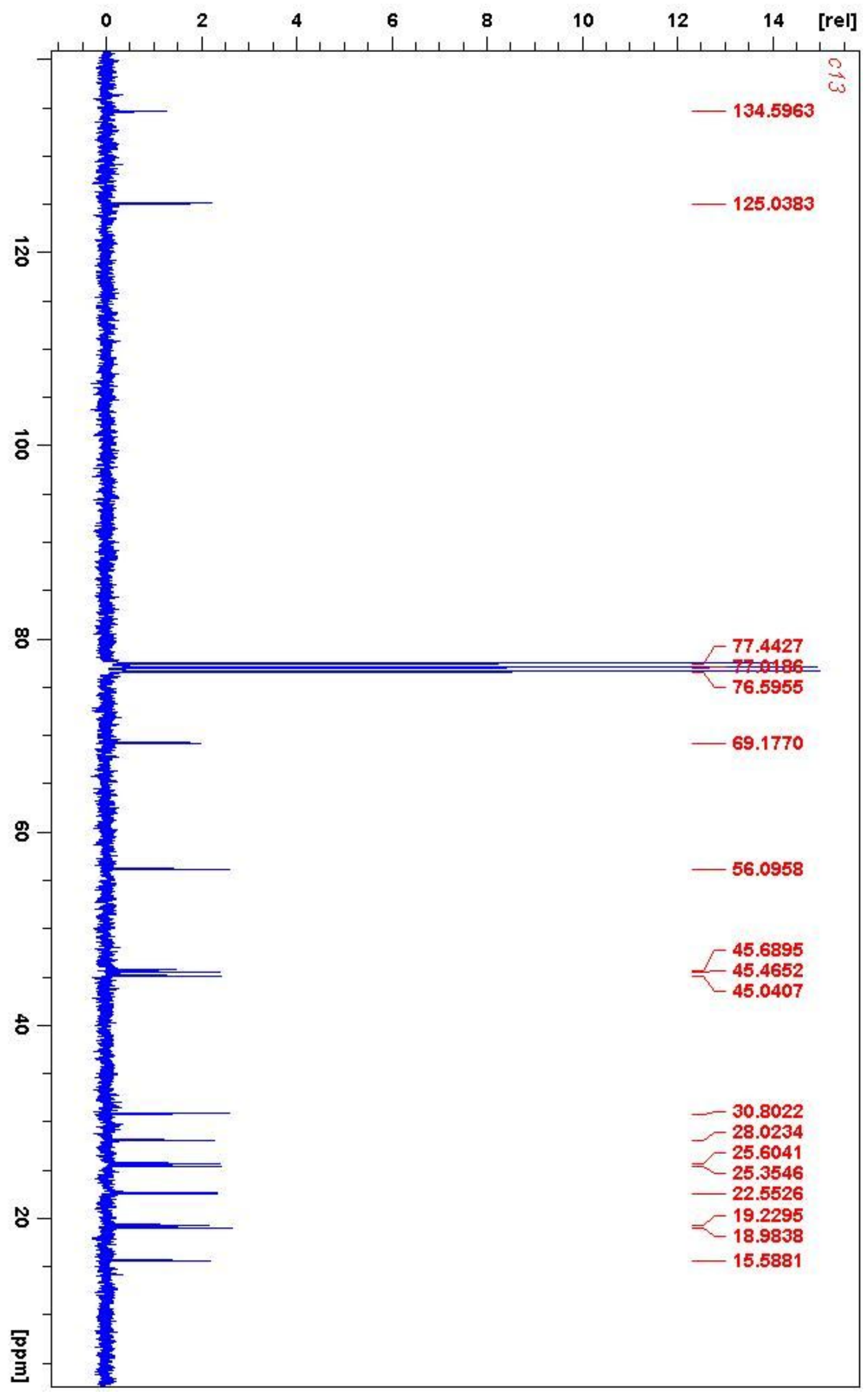

Figure S12. ${ }^{13} \mathrm{C}$ NMR spectrum of 2 in $\mathrm{CDCl}_{3}$ 


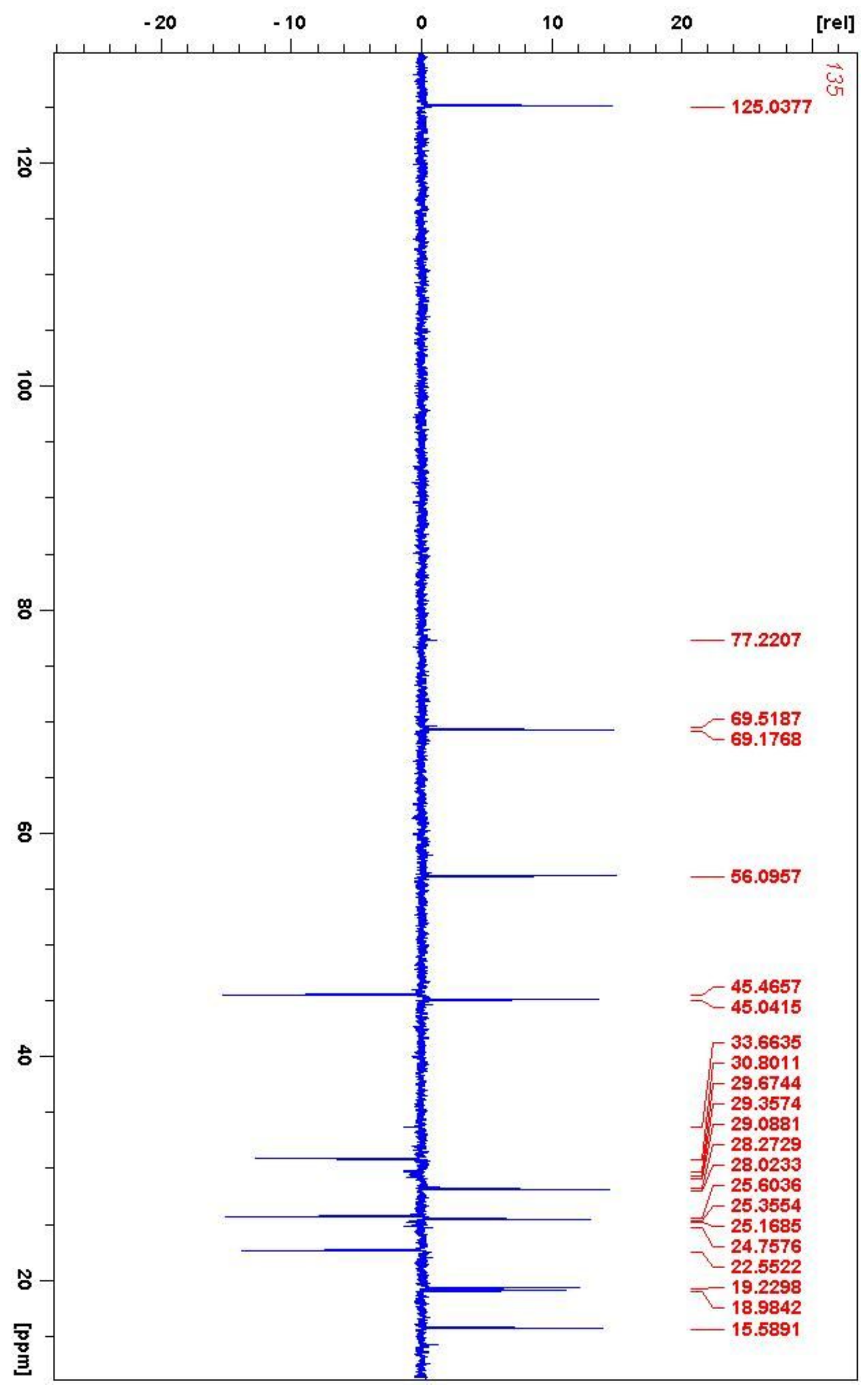

Figure S13. DEPT spectrum of 2 in $\mathrm{CDCl}_{3}$ 


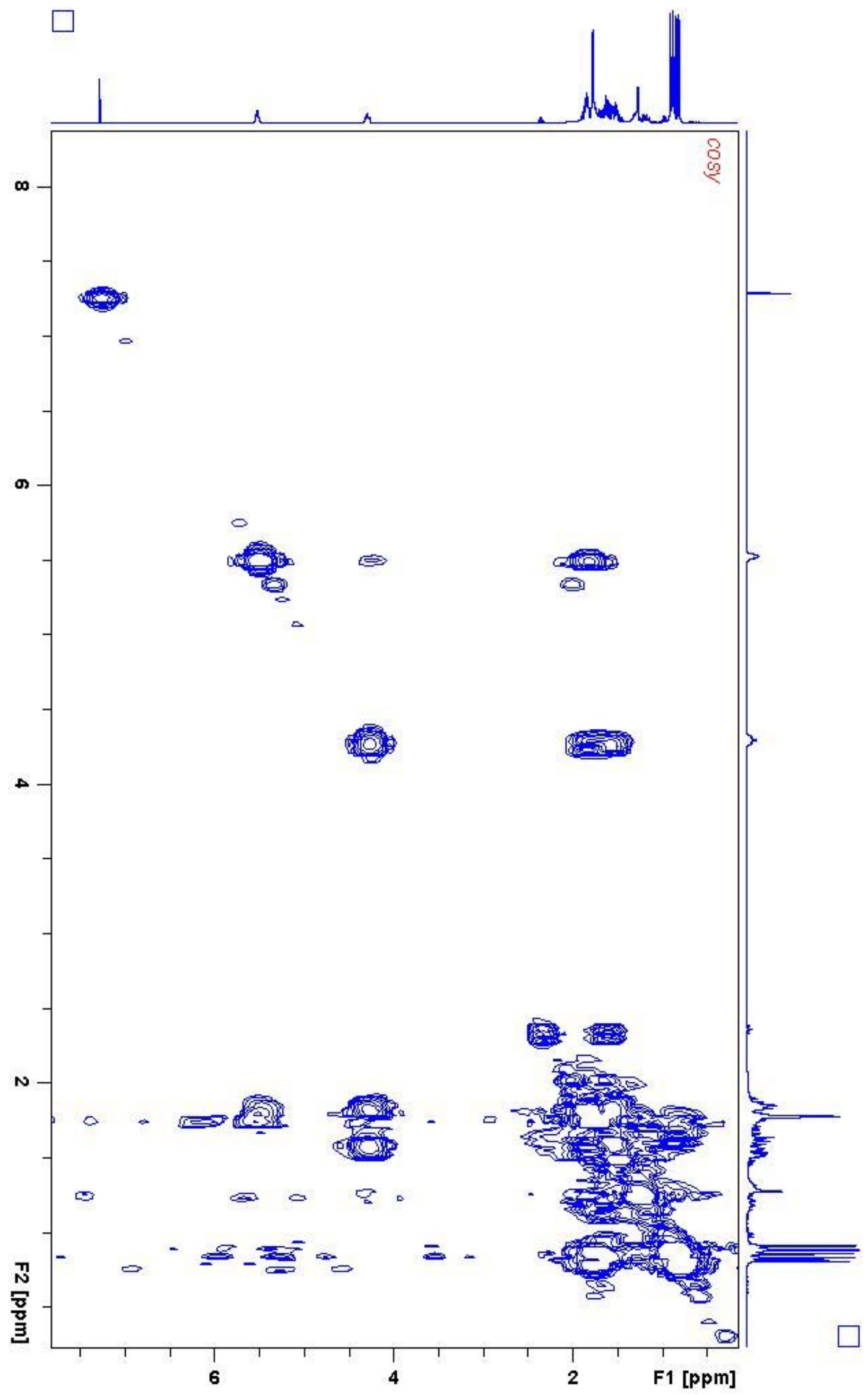

Figure S14. ${ }^{1} \mathrm{H}-{ }^{1} \mathrm{H}$ COSY spectrum of 2 in $\mathrm{CDCl}_{3}$ 


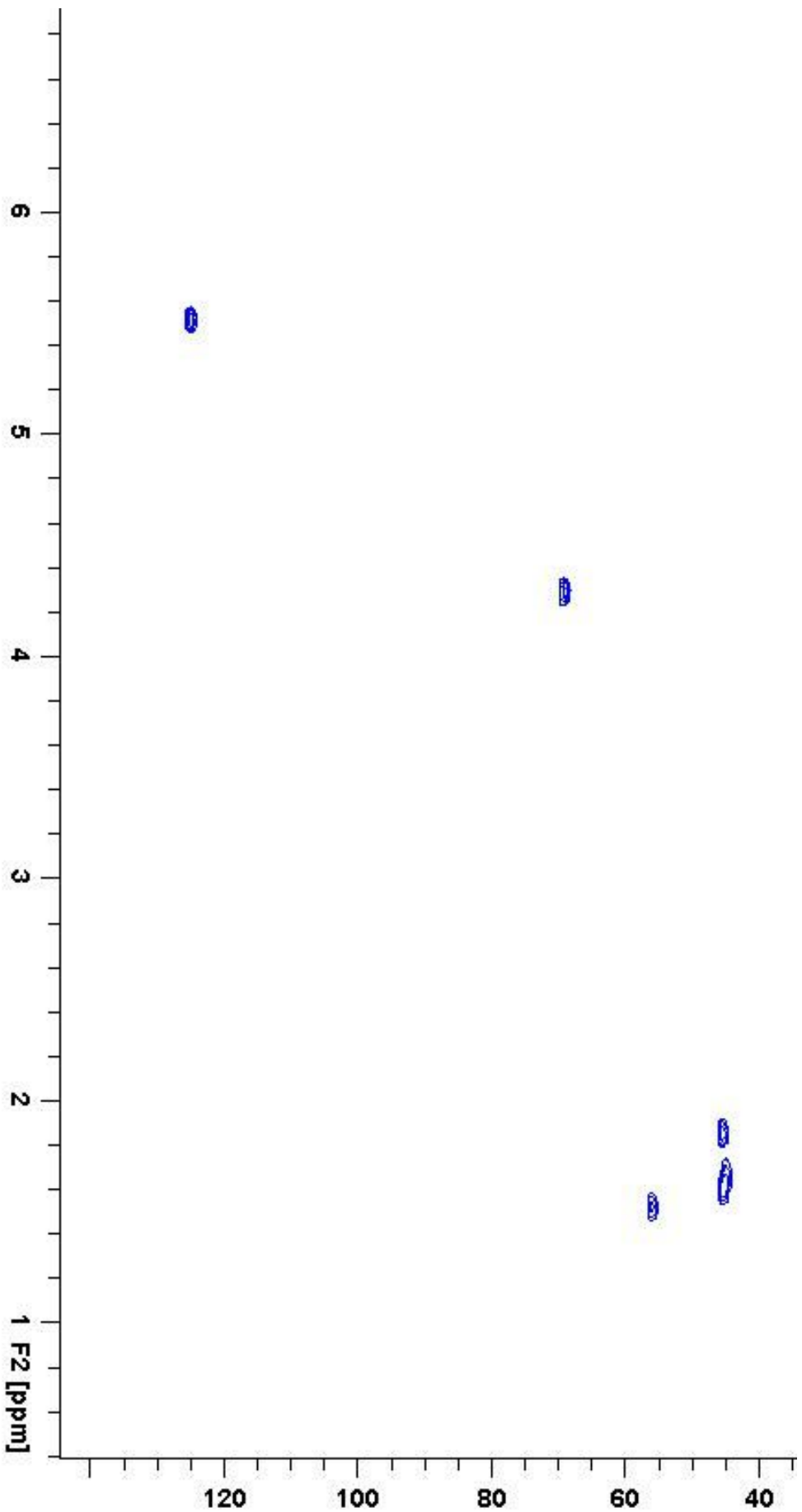

Figure S15. HSQC spectrum of 2 in $\mathrm{CDCl}_{3}$ 


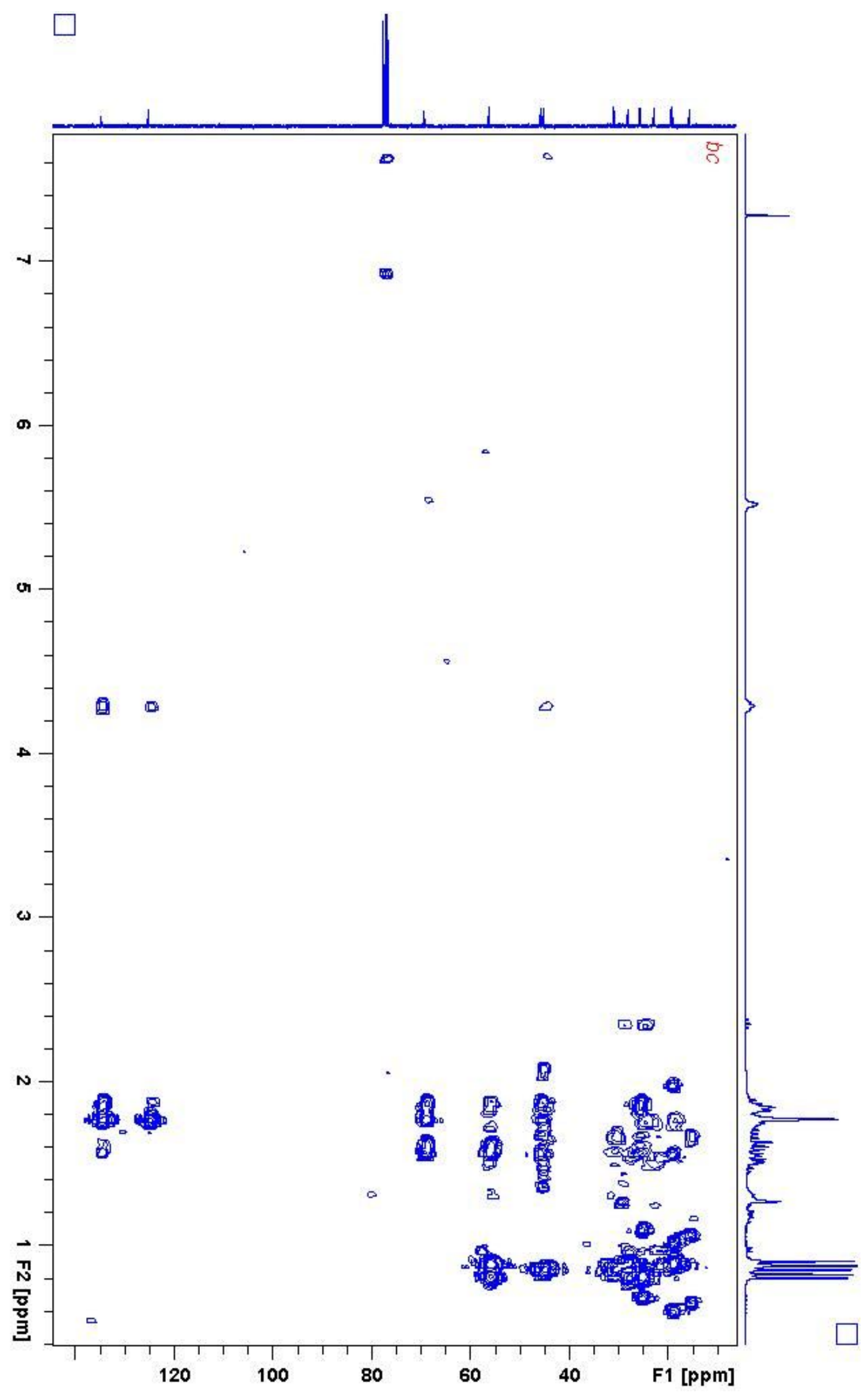

Figure S16. $\mathrm{HMBC}$ spectrum of 2 in $\mathrm{CDCl}_{3}$ 


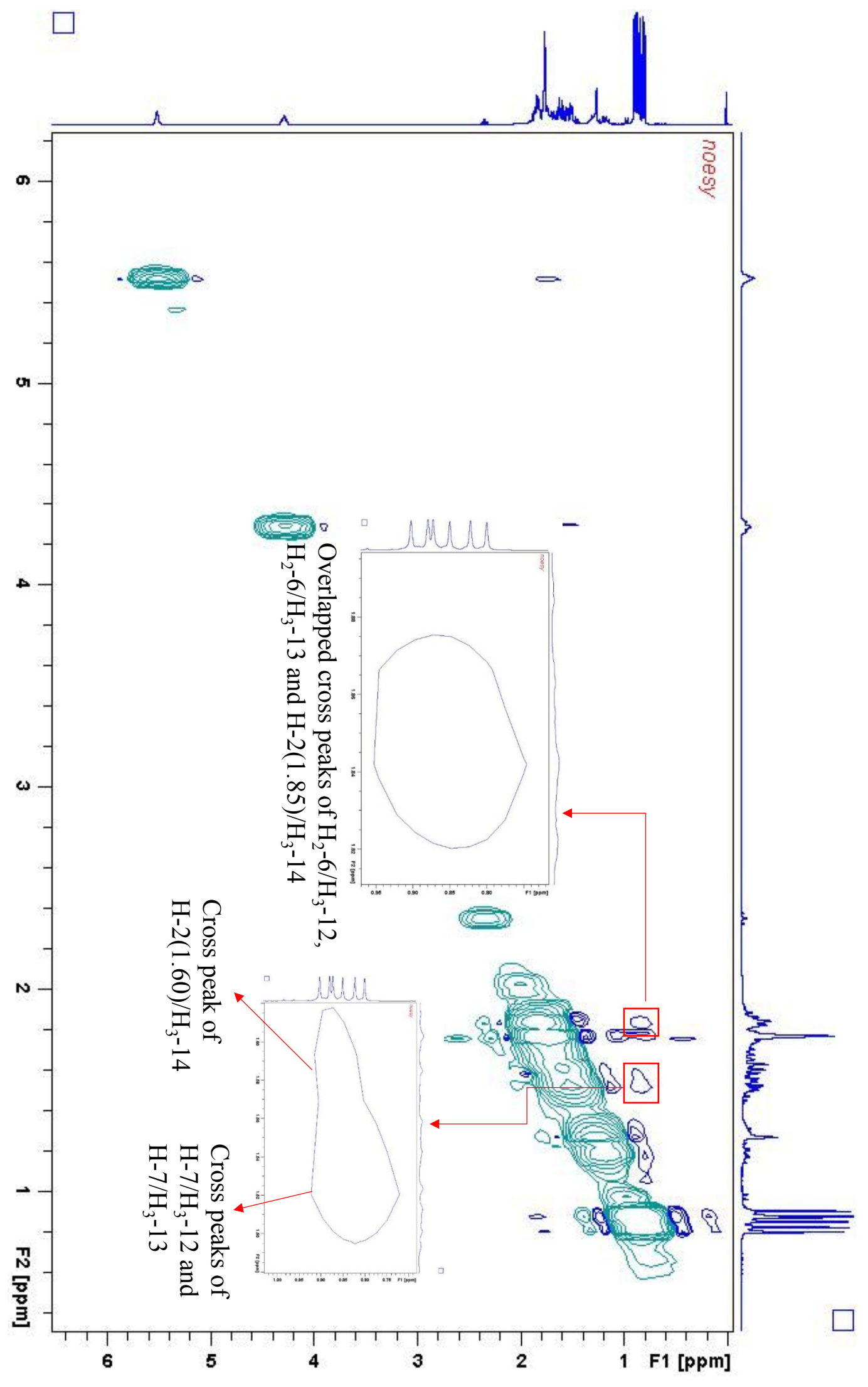

Figure S17. NOESY spectrum of 2 in $\mathrm{CDCl}_{3}$ 


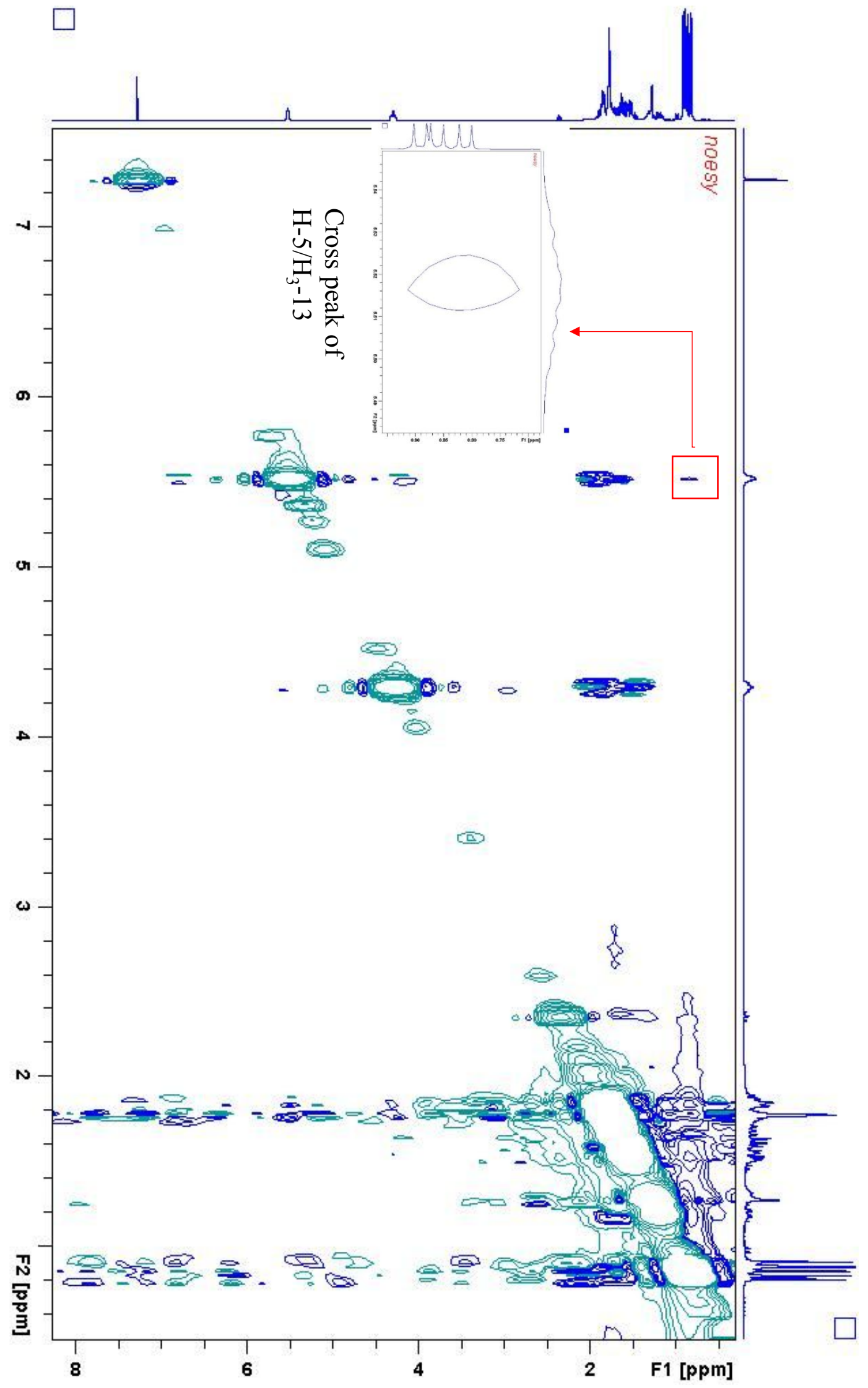

Figure S18. NOESY spectrum (lower contour level) of $\mathbf{2}$ in $\mathrm{CDCl}_{3}$ 

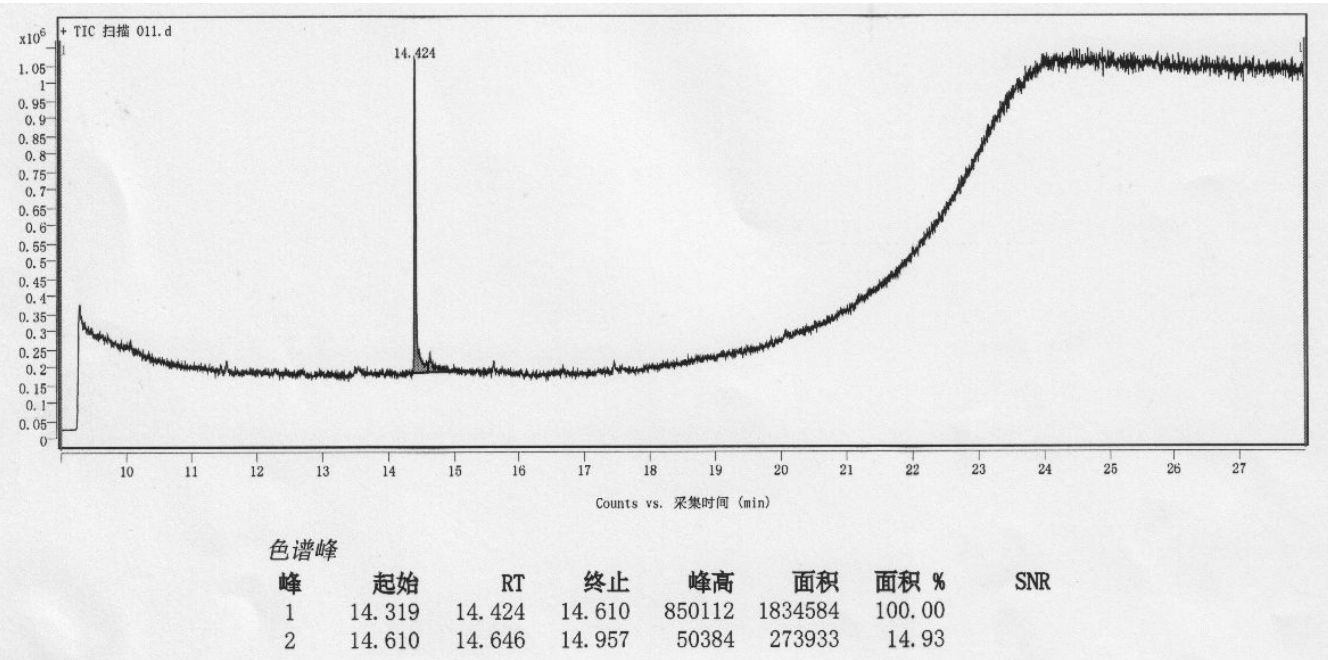

\section{样品谱图}

+ 扫描 $(\mathrm{rt}: 14.422 \mathrm{~min})$ 扣除

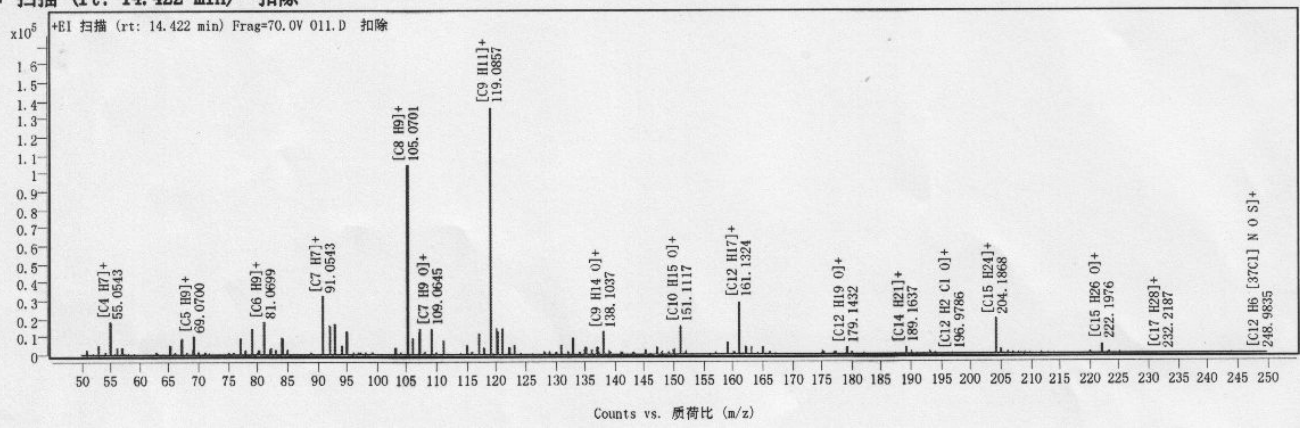

Figure S19. The HREIMS spectrum of $\mathbf{3}$

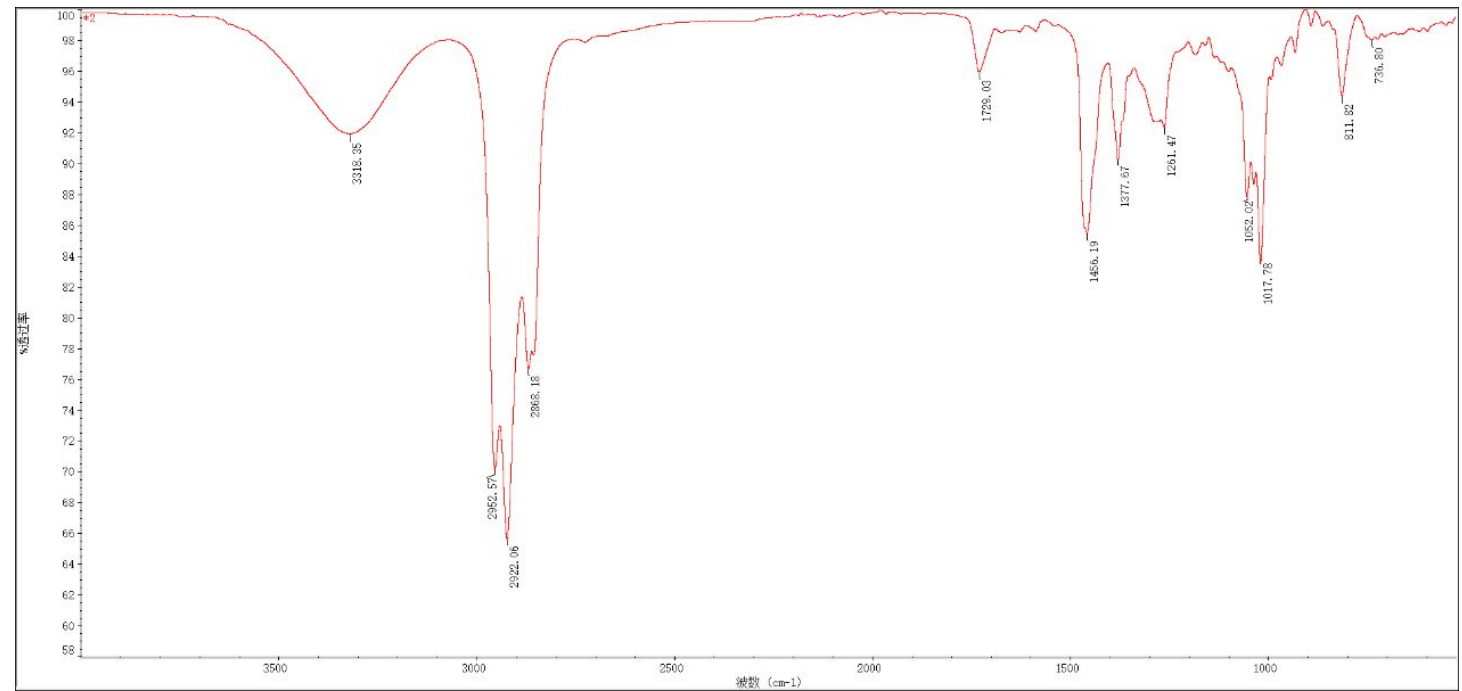

Figure S20. IR spectrum of $\mathbf{3}(\mathrm{KBr})$ 


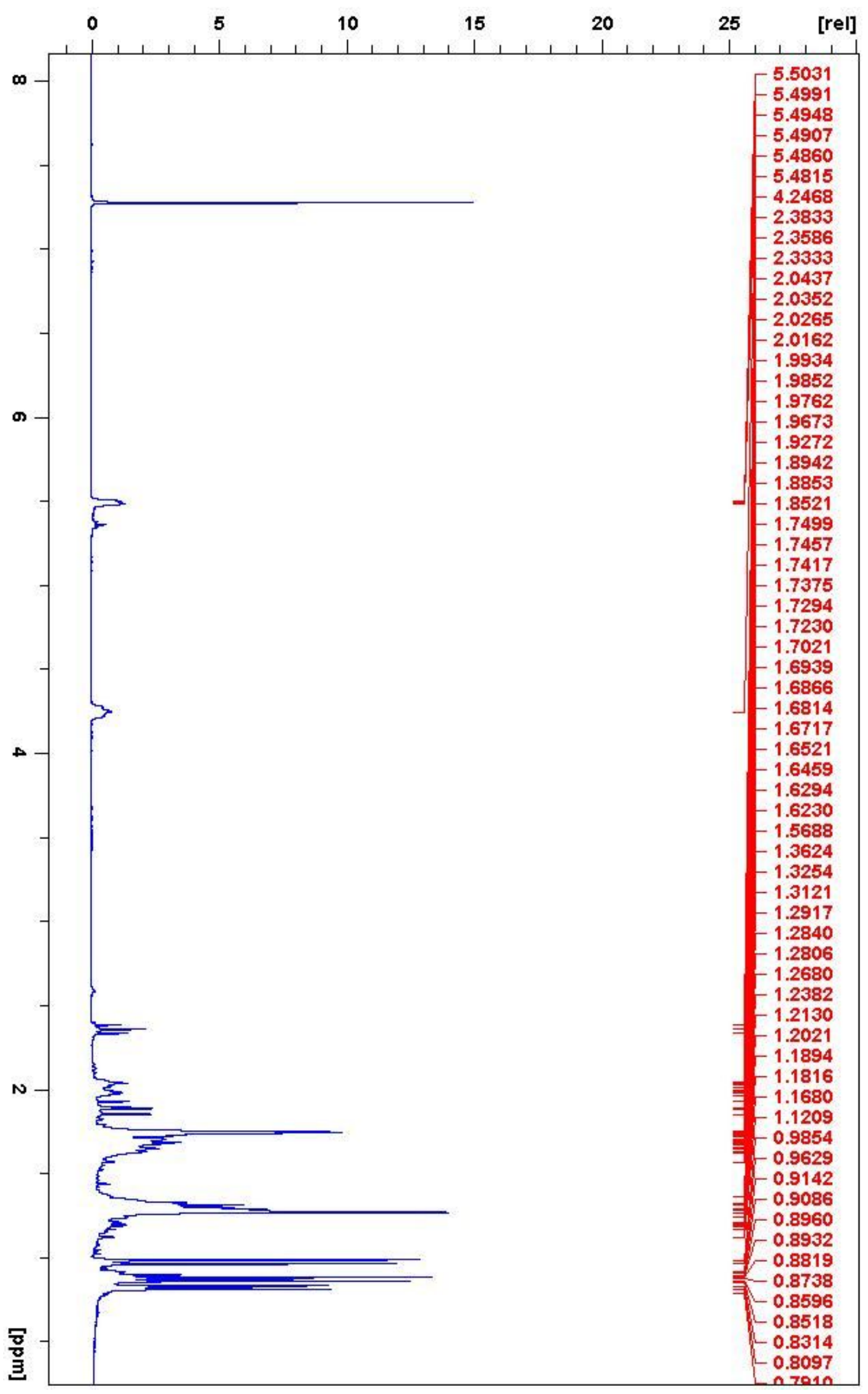

Figure S21. ${ }^{1} \mathrm{H}$ NMR spectrum of 3 in $\mathrm{CDCl}_{3}$ 


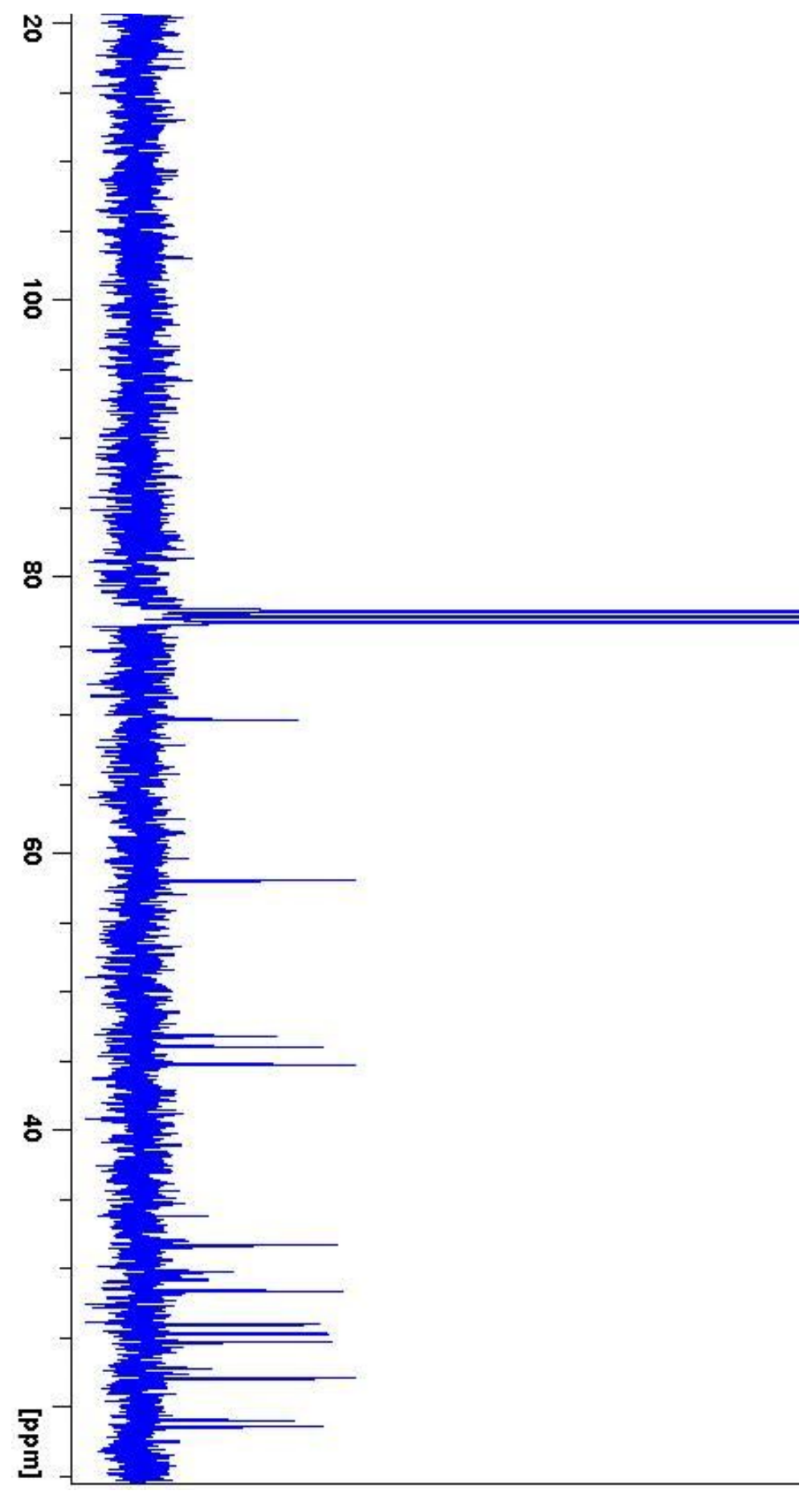

Figure S22. ${ }^{13} \mathrm{C}$ NMR spectrum of $\mathbf{3}$ in $\mathrm{CDCl}_{3}$ 


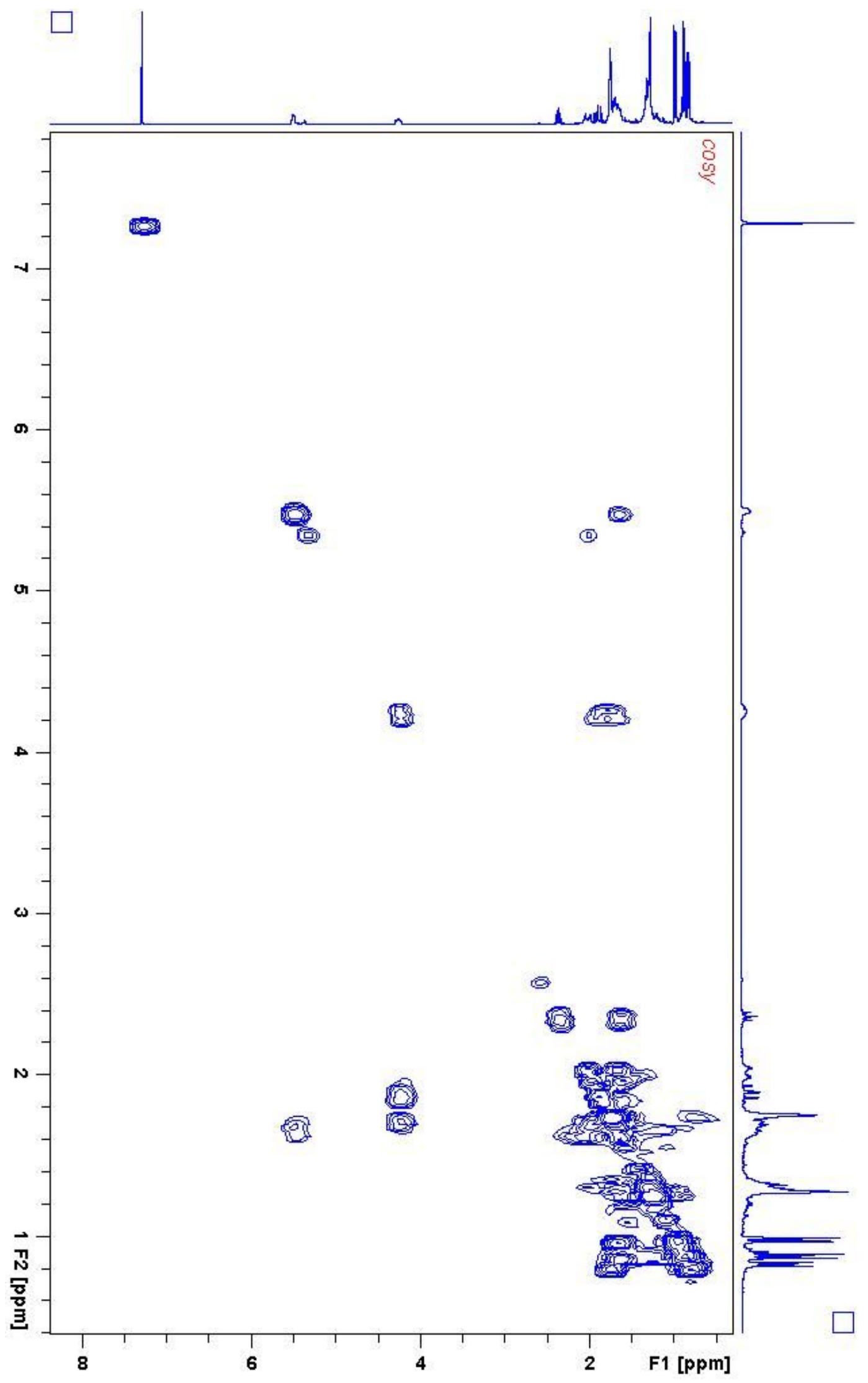

Figure S23. ${ }^{1} \mathrm{H}-{ }^{1} \mathrm{H}$ COSY spectrum of $\mathbf{3}$ in $\mathrm{CDCl}_{3}$ 


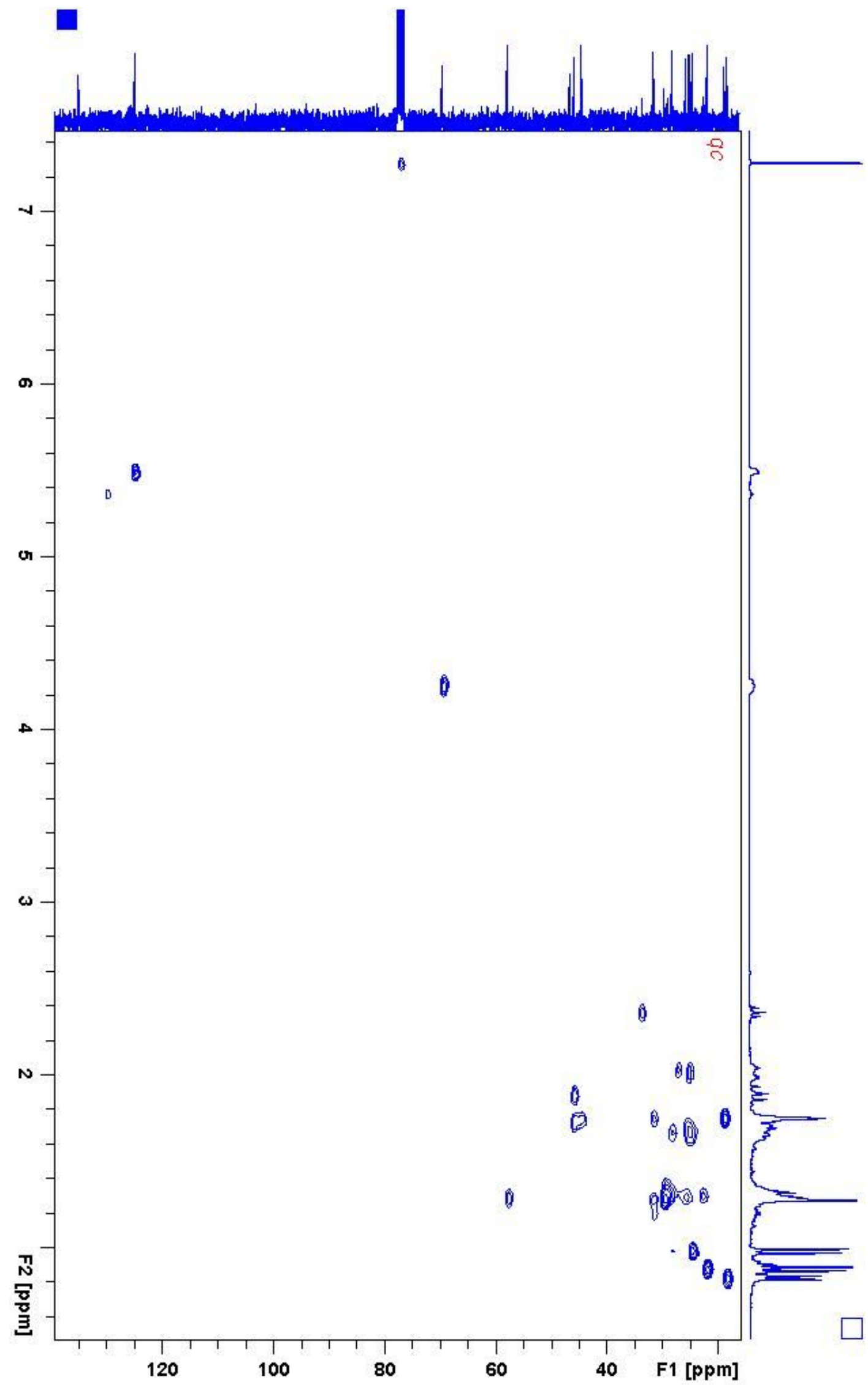

Figure S24. HSQC spectrum of 3 in $\mathrm{CDCl}_{3}$ 


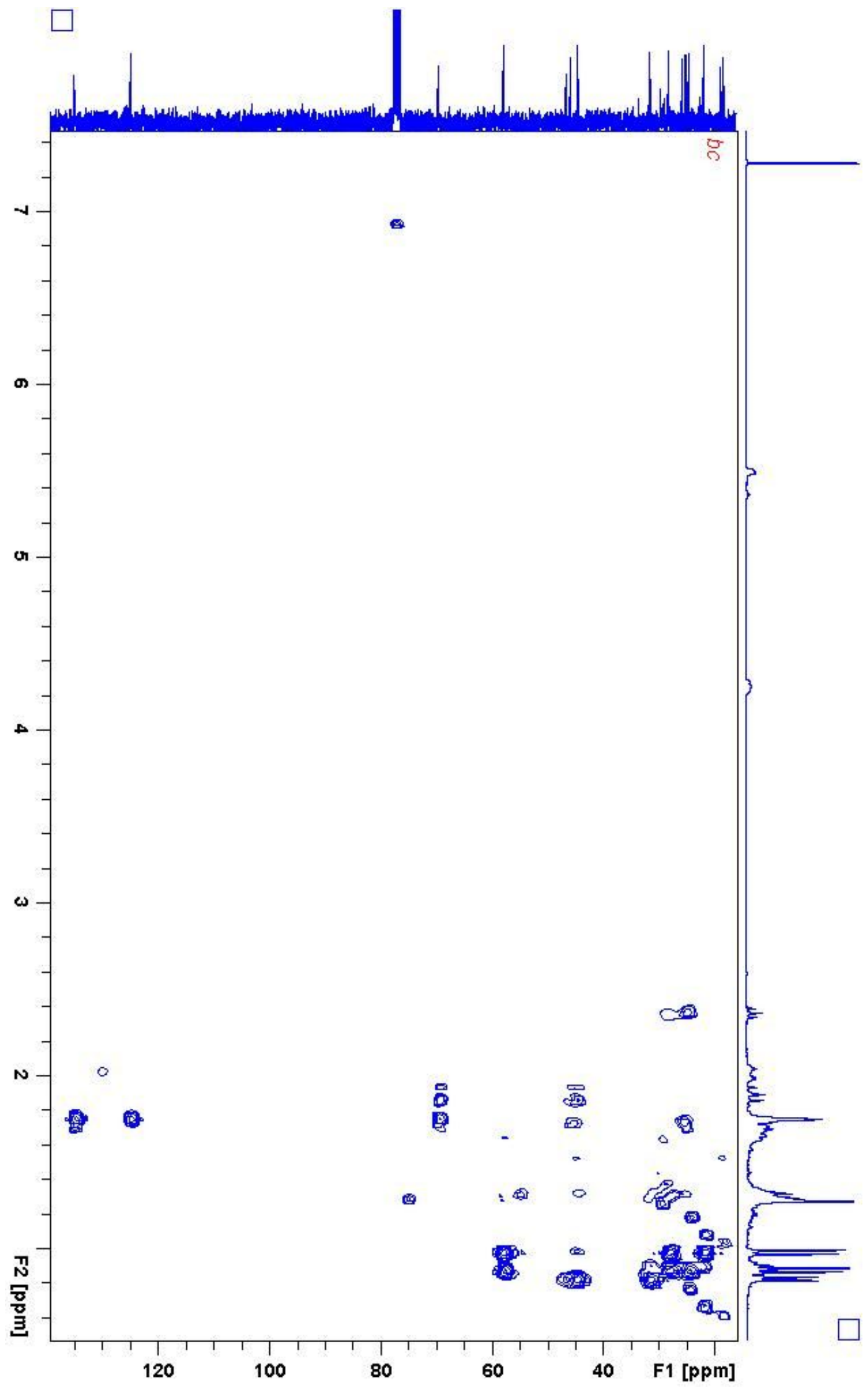

Figure S25. $\mathrm{HMBC}$ spectrum of $\mathbf{3}$ in $\mathrm{CDCl}_{3}$ 


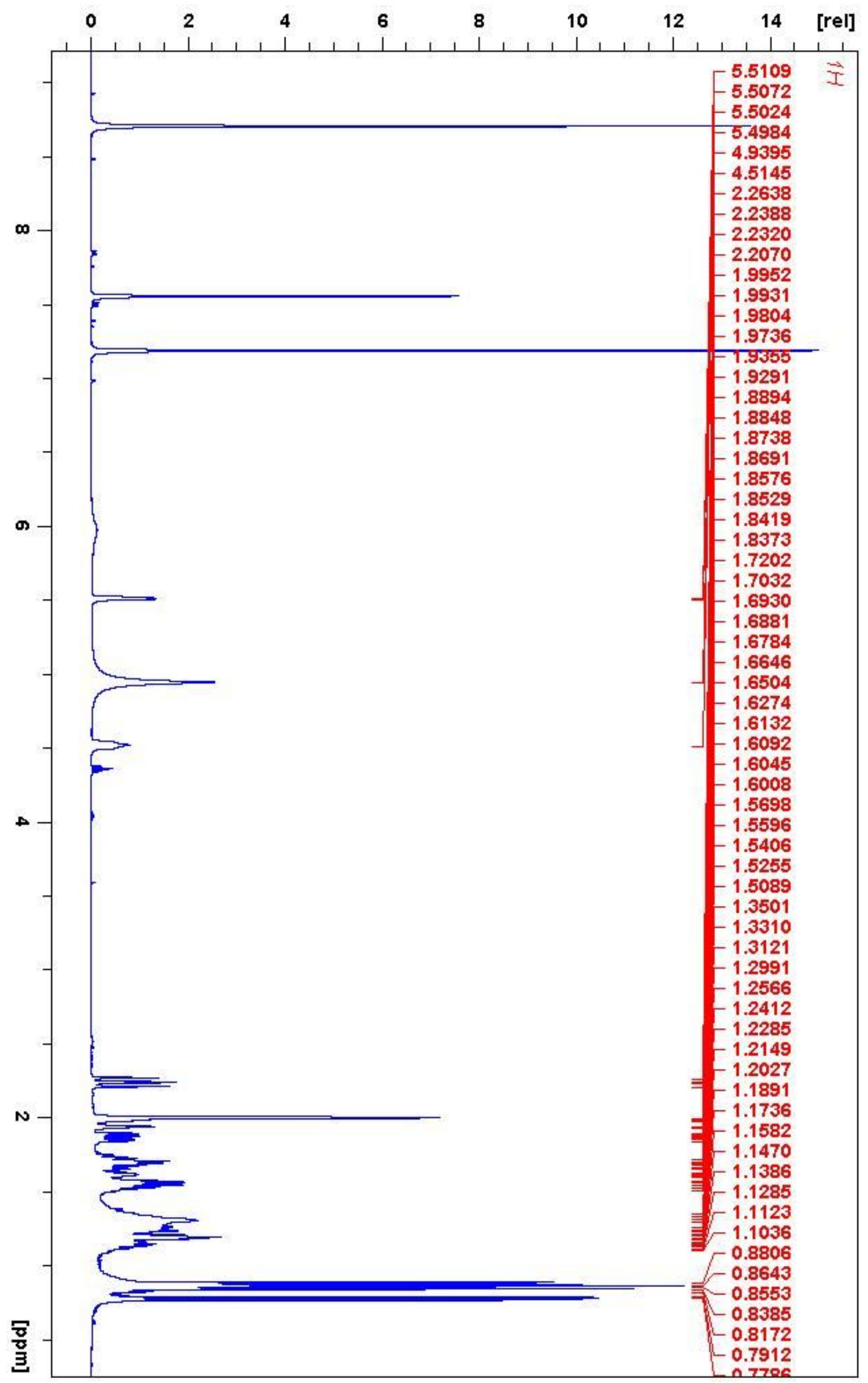

Figure S26. ${ }^{1} \mathrm{H}$ NMR spectrum of $\mathbf{3}$ in pyridine- $d_{5}$ 


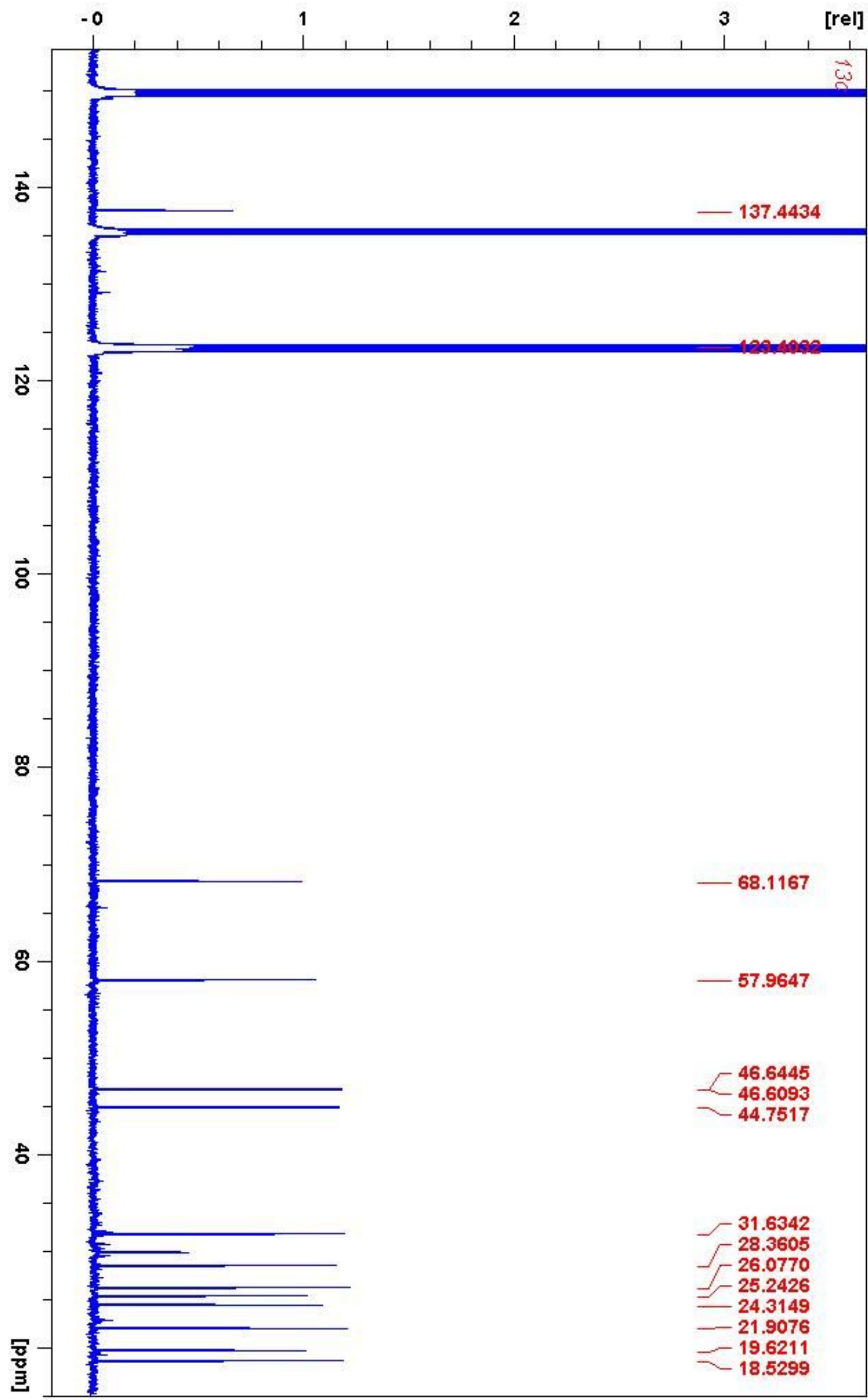

Figure S27. ${ }^{13} \mathrm{C}$ NMR spectrum of $\mathbf{3}$ in pyridine- $d_{5}$ 


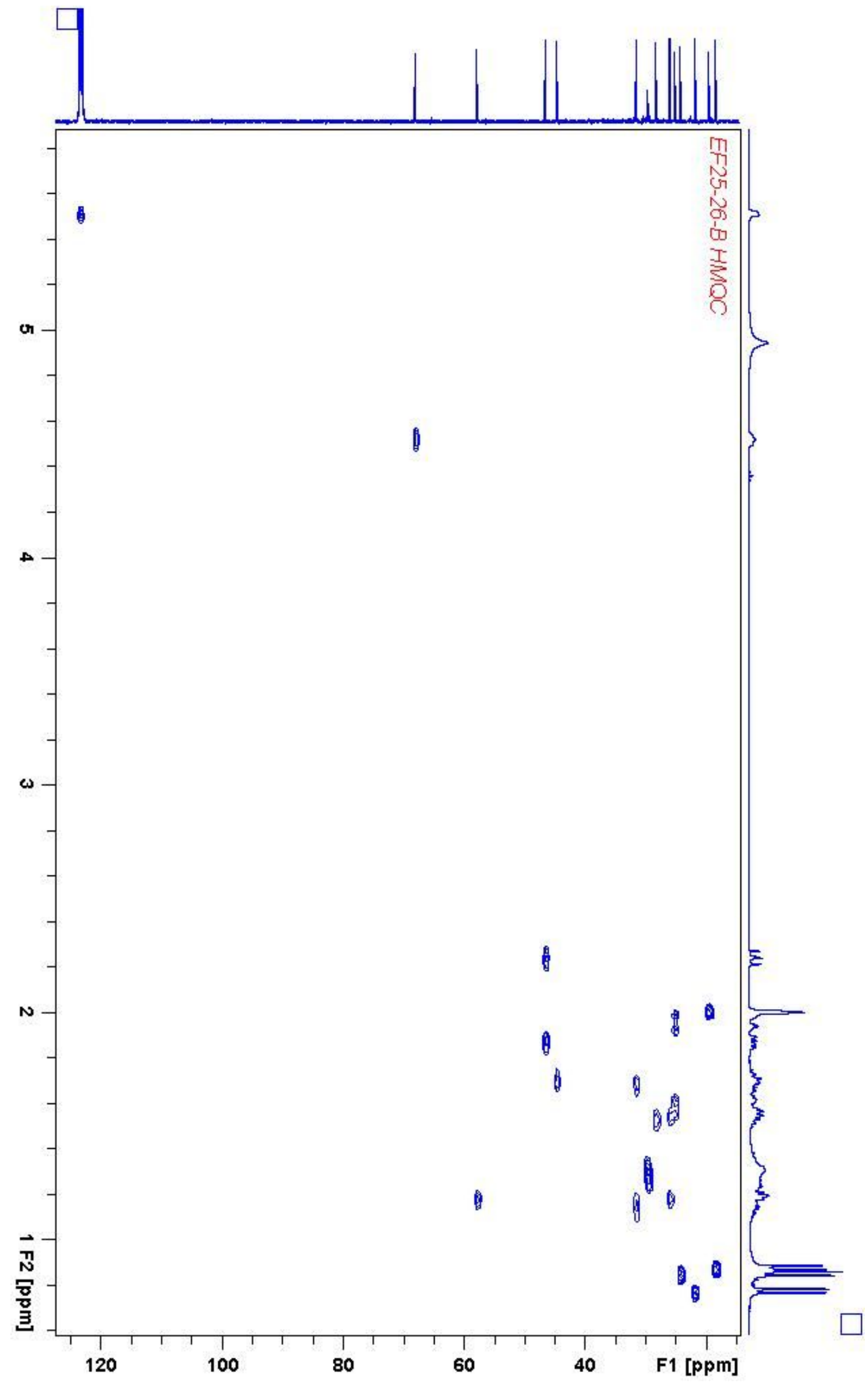

Figure S28. HSQC spectrum of $\mathbf{3}$ in pyridine- $d_{5}$ 


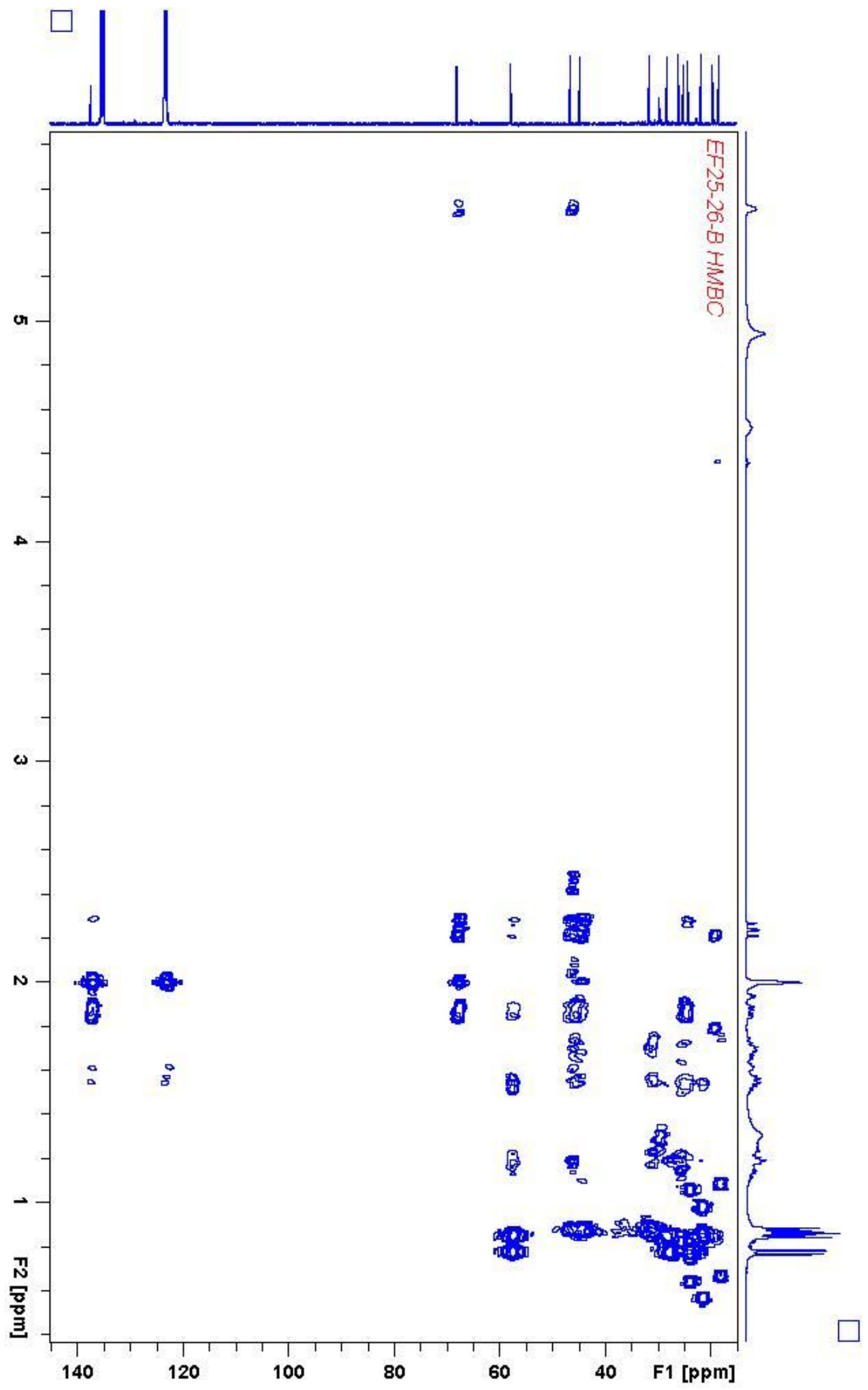

Figure S29. HMBC spectrum of $\mathbf{3}$ in pyridine- $d_{5}$ 


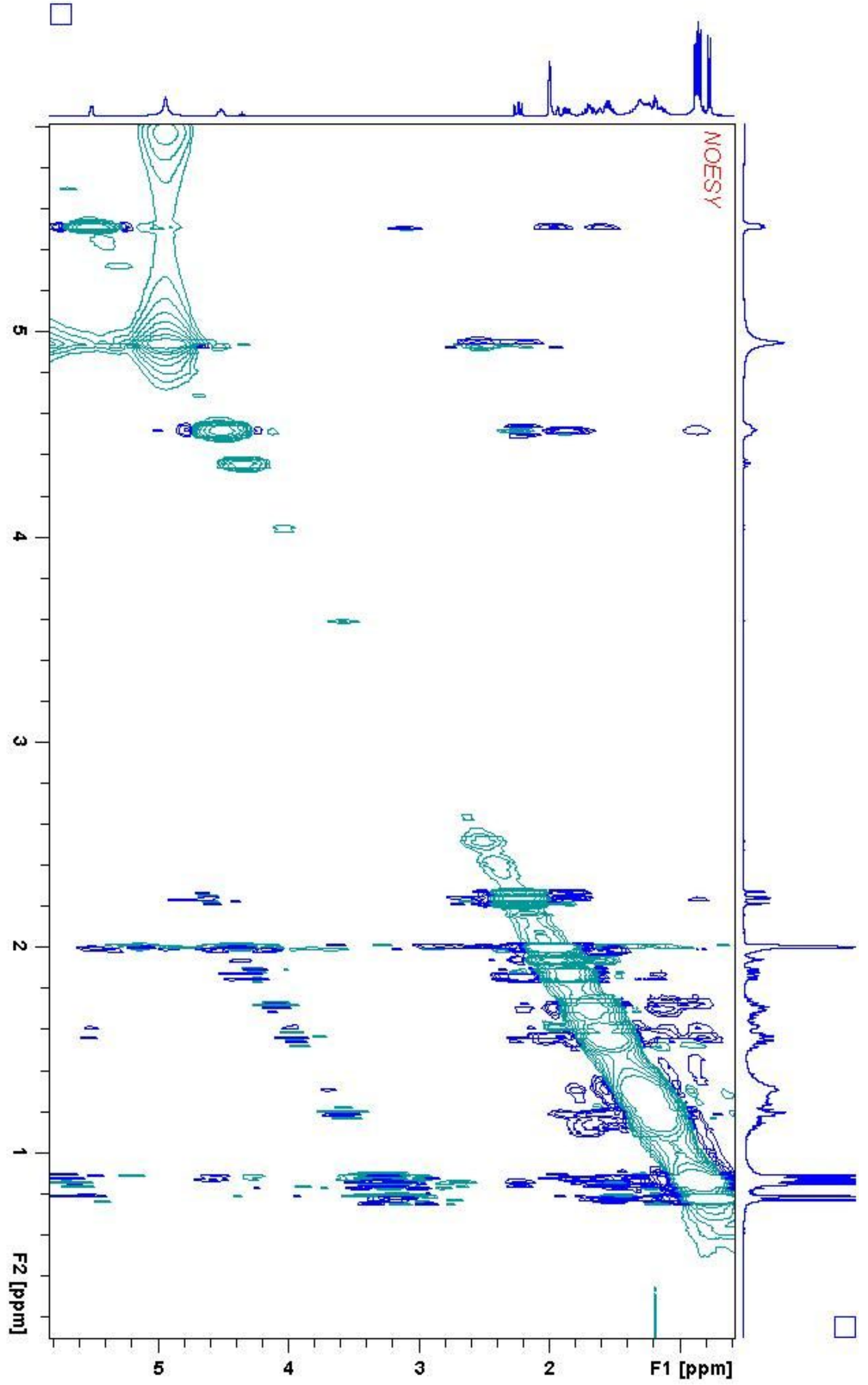

Figure S30. NOESY spectrum of $\mathbf{3}$ in pyridine- $d_{5}$ 

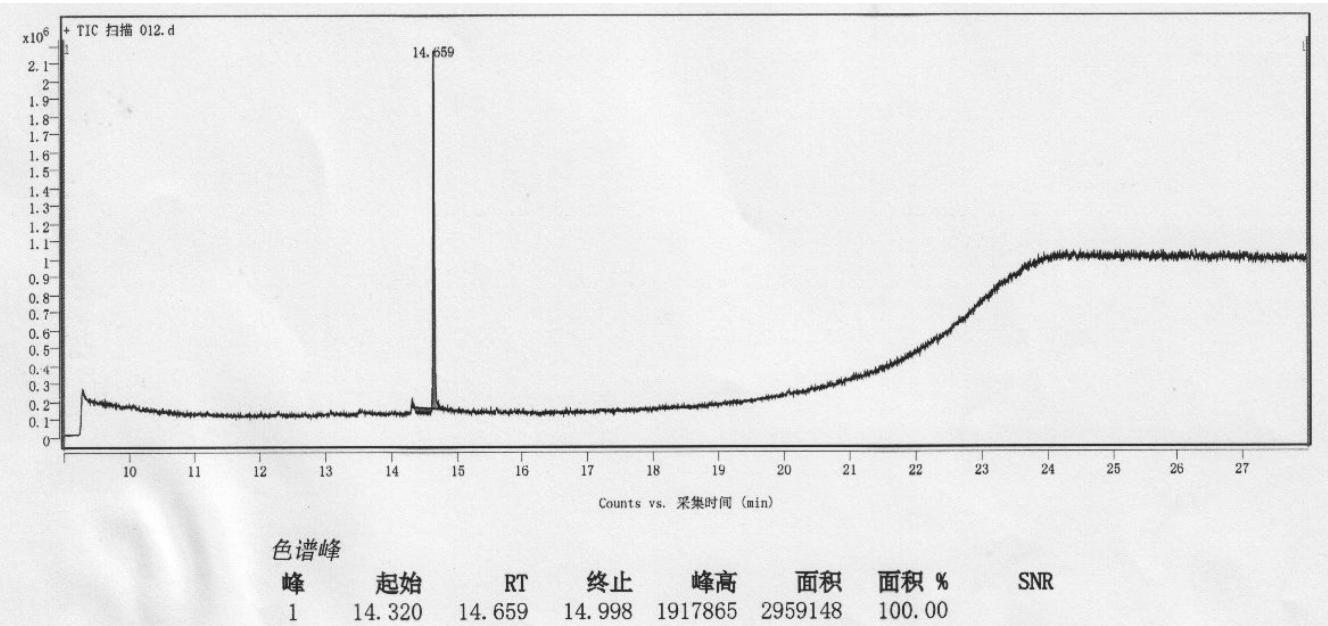

样品谱图

+ 扫描 $(\mathrm{rt}: 14.657 \mathrm{~min})$ 扣除

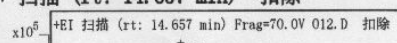

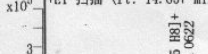

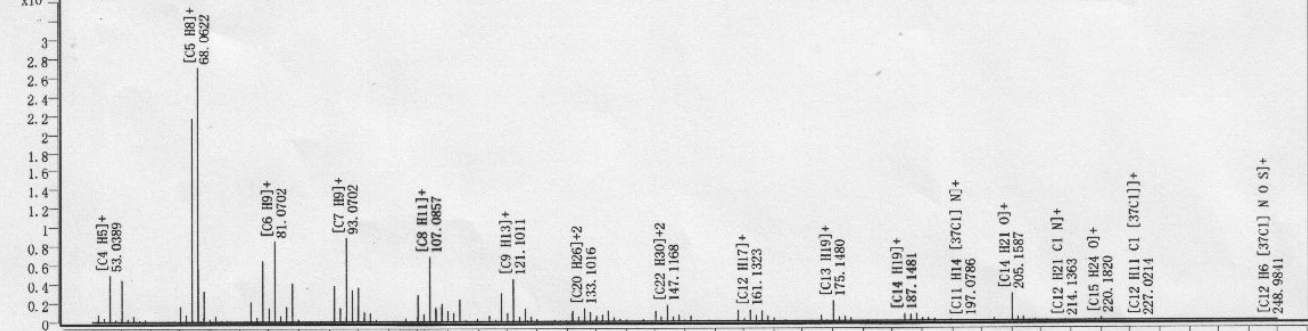

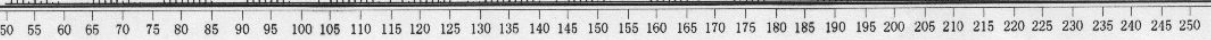
Counts vs. 秀附比 $(\mathbf{m} / \mathbf{z})$

Figure S31. The HREIMS spectrum of $\mathbf{4}$

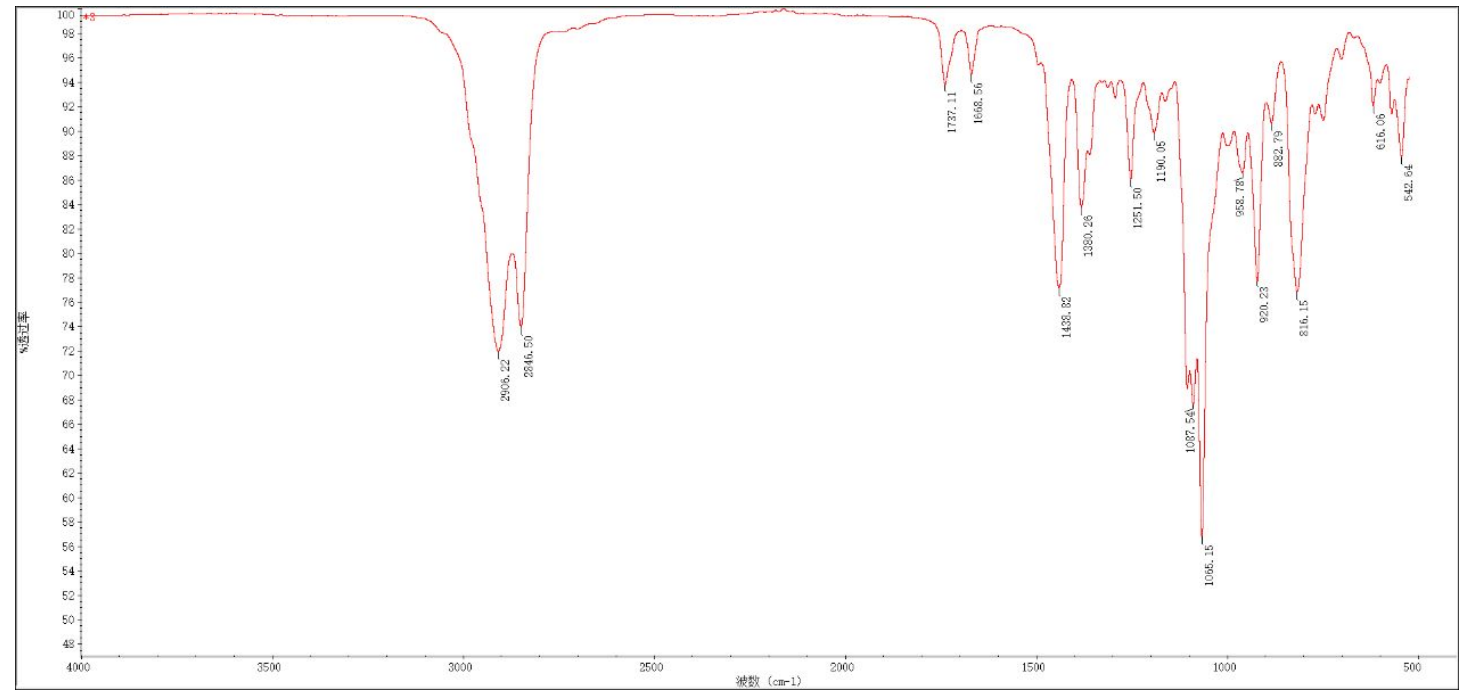

Figure S32. IR spectrum of $4(\mathrm{KBr})$ 


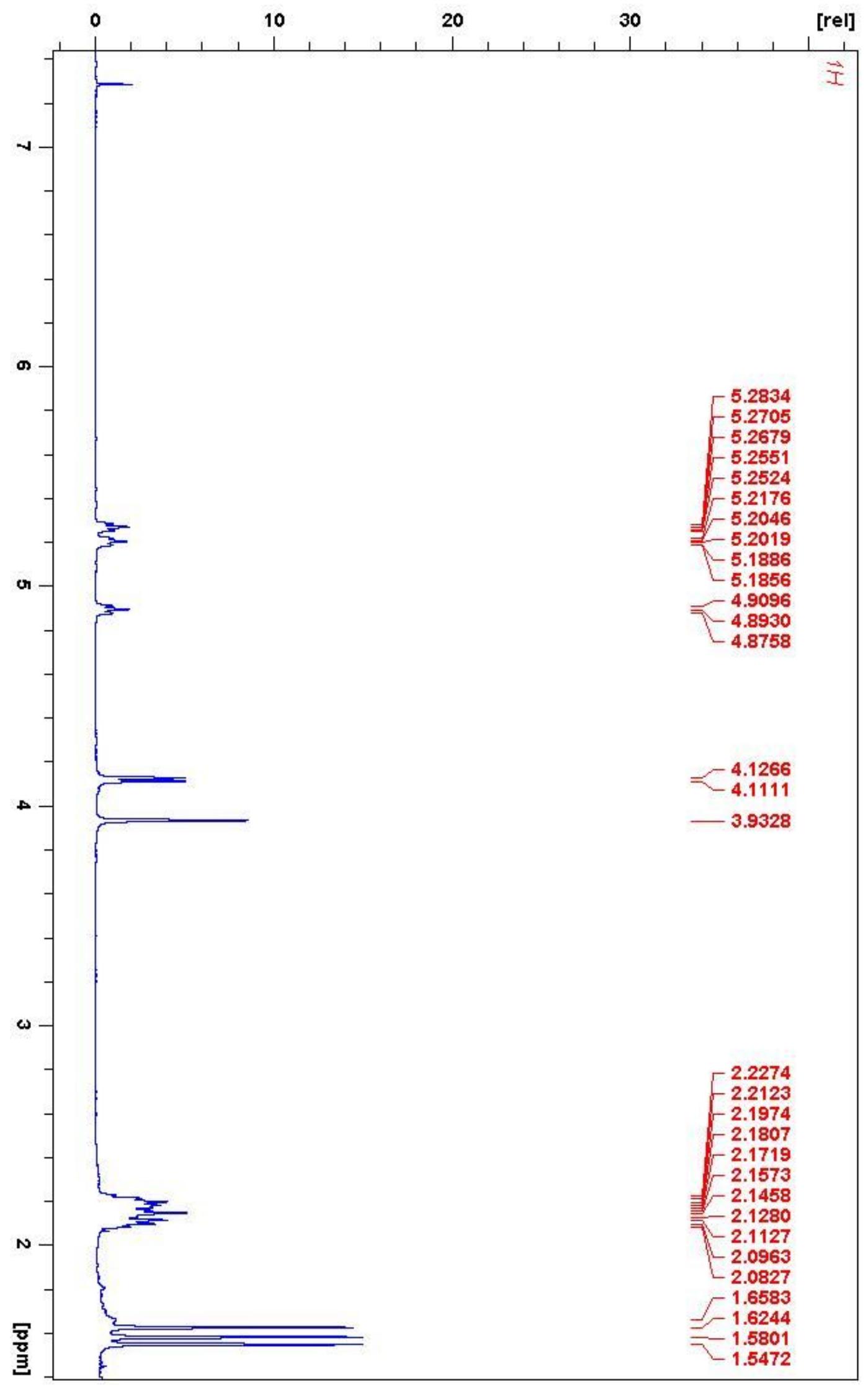

Figure S33. ${ }^{1} \mathrm{H}$ NMR spectrum of 4 in $\mathrm{CDCl}_{3}$ 


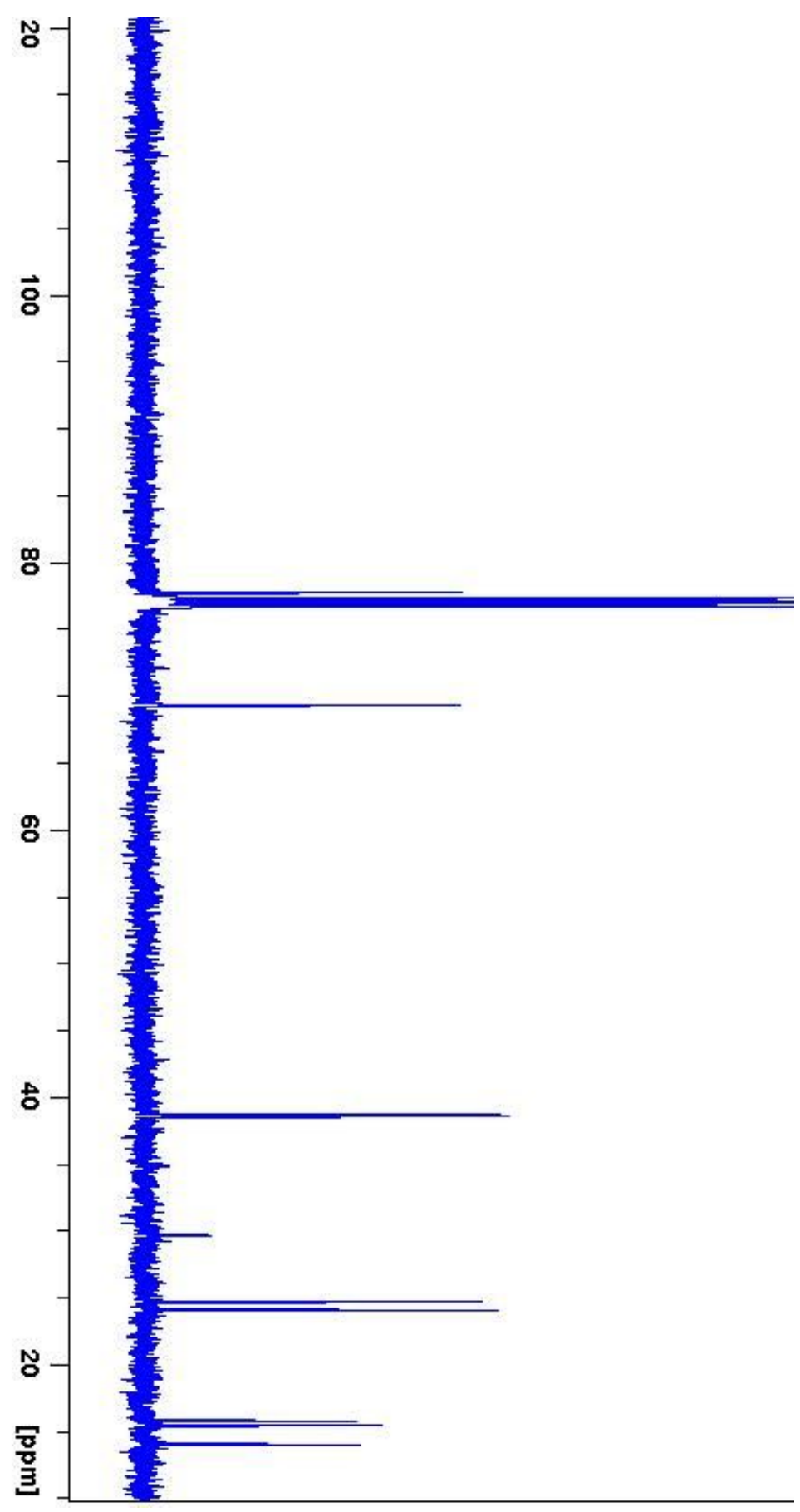

Figure S34. ${ }^{13} \mathrm{C}$ NMR spectrum of 4 in $\mathrm{CDCl}_{3}$ 


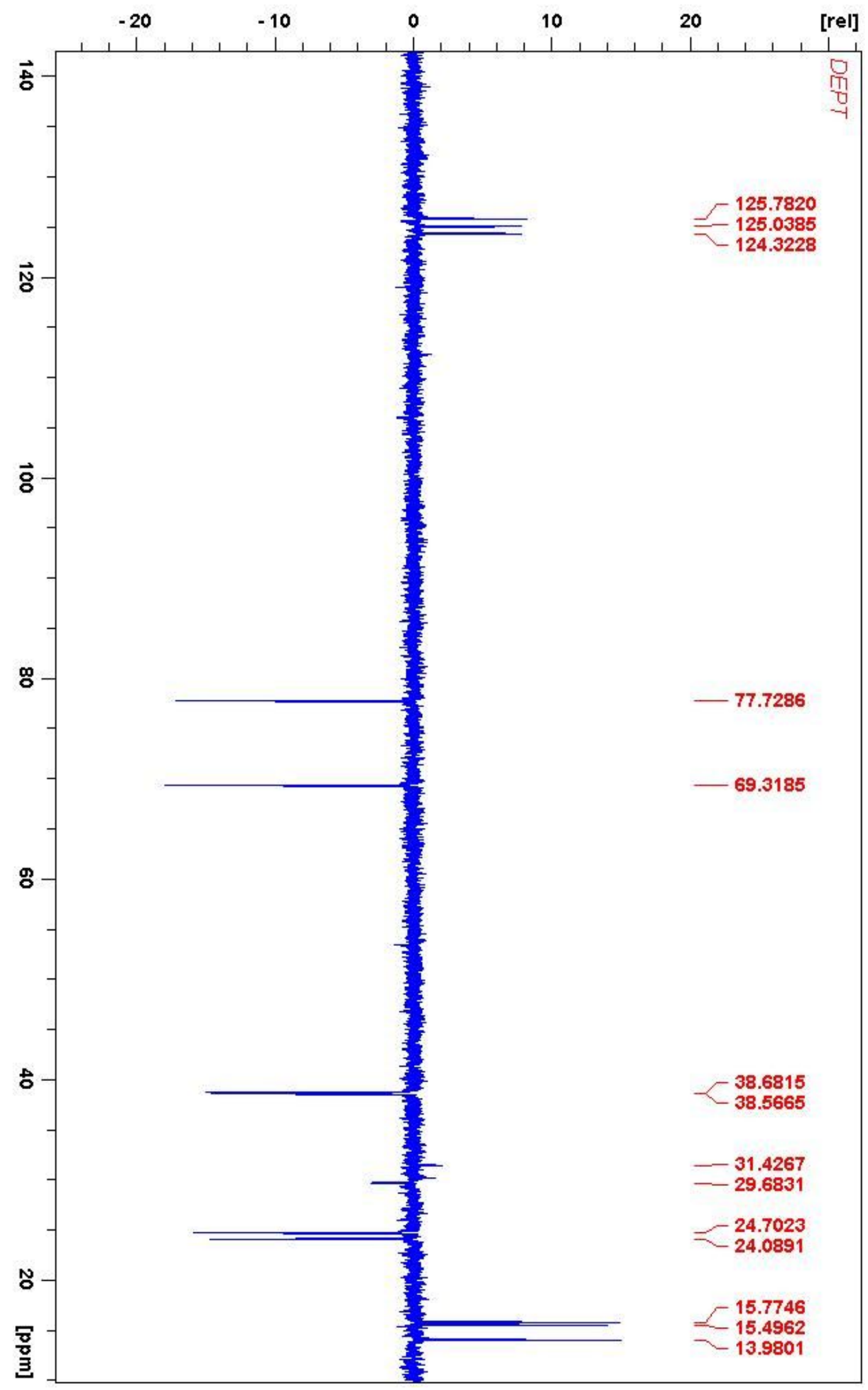

Figure S35. DEPT spectrum of 4 in $\mathrm{CDCl}_{3}$ 


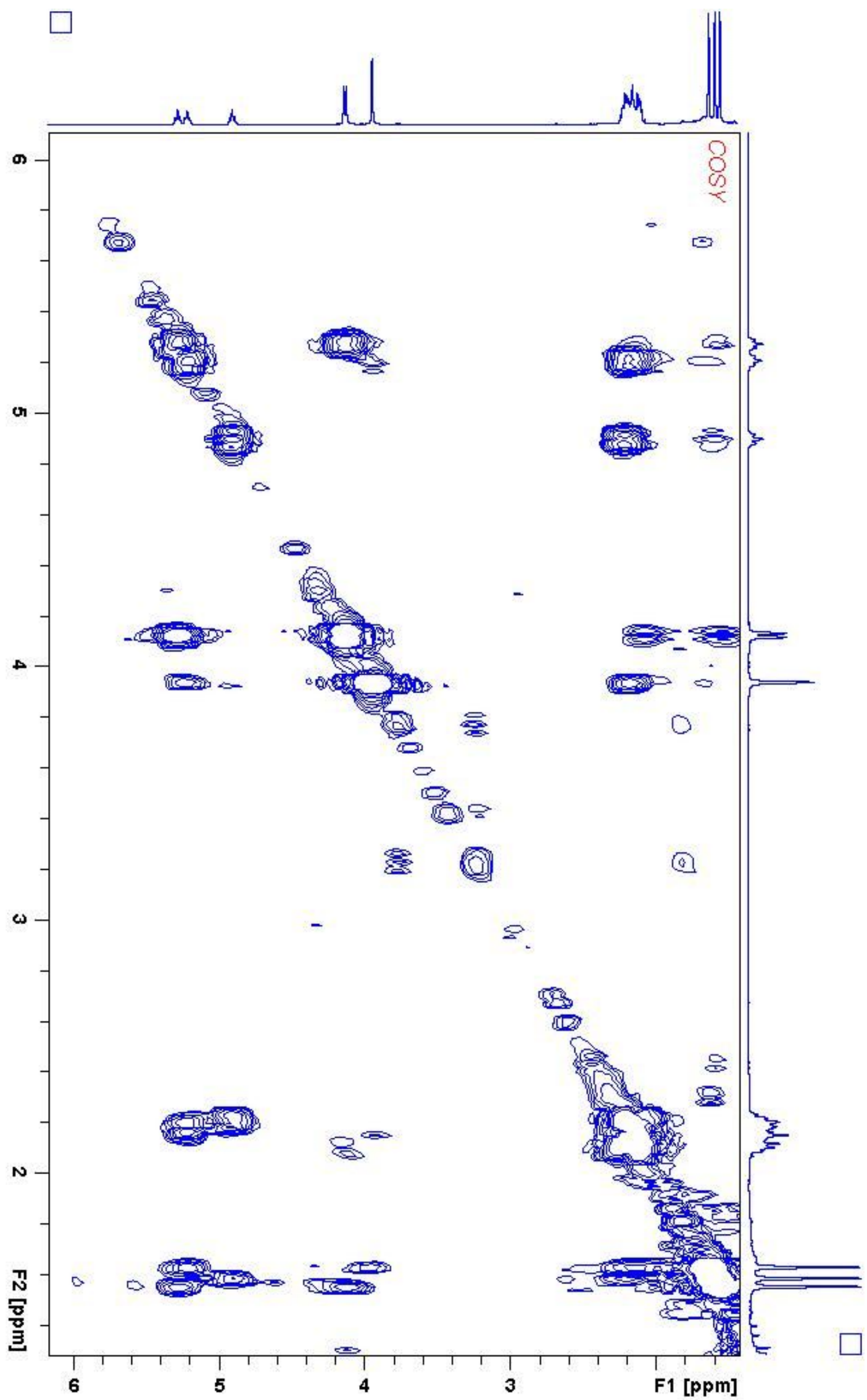

Figure S36. ${ }^{1} \mathrm{H}-{ }^{1} \mathrm{H}$ COSY spectrum of 4 in $\mathrm{CDCl}_{3}$ 


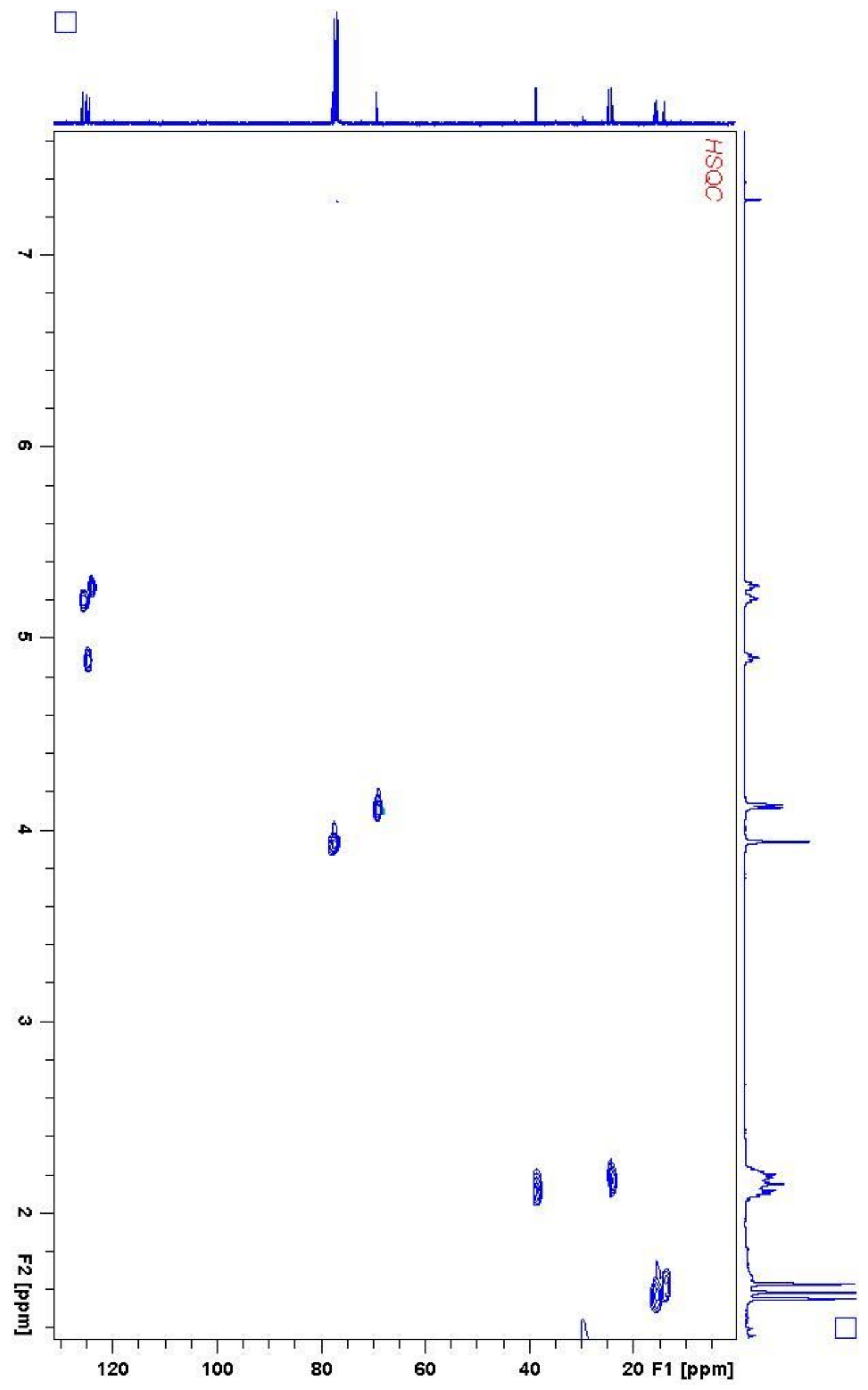

Figure S37. HSQC spectrum of 4 in $\mathrm{CDCl}_{3}$ 


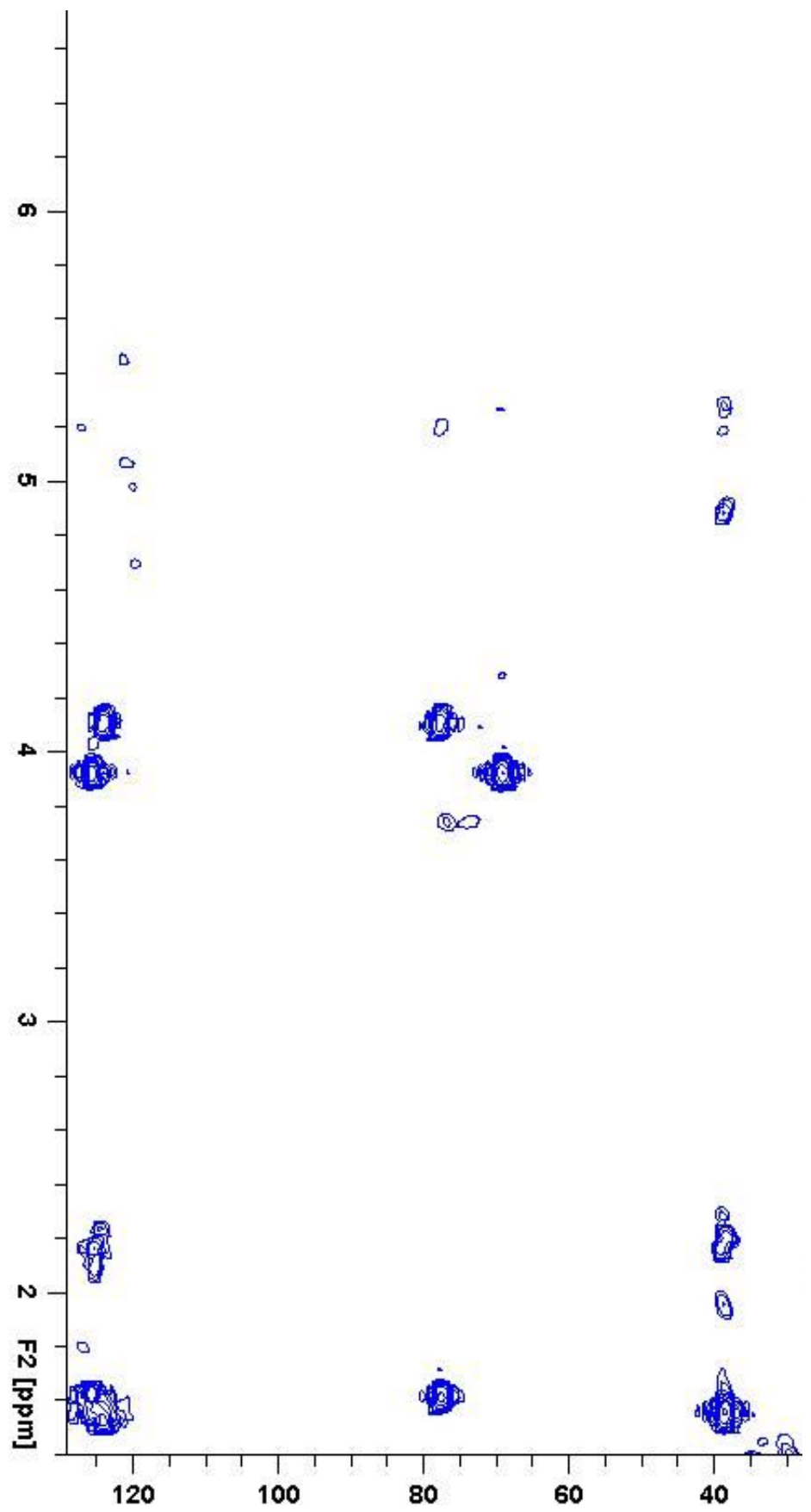

Figure S38. $\mathrm{HMBC}$ spectrum of 4 in $\mathrm{CDCl}_{3}$ 


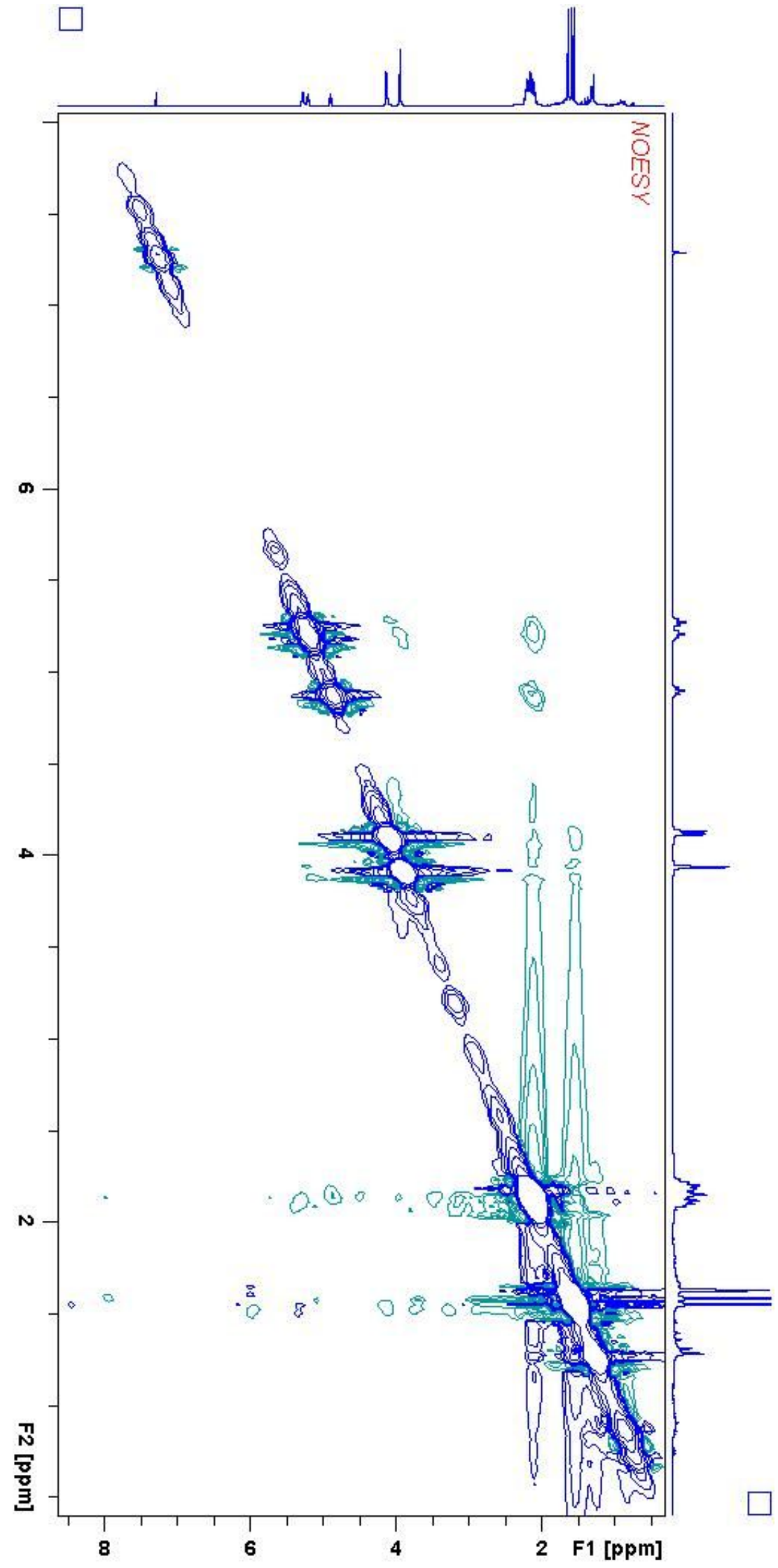

Figure S39. NOESY spectrum of 4 in $\mathrm{CDCl}_{3}$ 
Recombinant $\boldsymbol{E} \boldsymbol{C} \boldsymbol{C A S}$ gene (blue: the sequence deriving from pCold TF vector; black: $E f C A S$ gene)

atgaatcatcatcatcatcatcatatcgaaggtaggcatatgatggcaaaagtagagcatgatgttcttcgtggtgcttccaaaaacgttcctaatg gcatttggggttatagccttgcttcaatttccccatcacttgatttggaactggaatcacacggcgaggaggtagaagcgattaaagaaaaagtg aaggatatgctcattgaatctgcaaaagaattgacttgtaacattgaattcatcaatttattatgccgtcttggcgtatcctattattttgaggtcgaaa tcgataatcaacttgatcatattttcaataatatcctcccagacattgtagaagataatgactatgatctttctactctggcaactttatttcgagtcttg cgacaacatggacacaaaatgacttctgatgtgtttaagaaattcaagagcagagatggagaattcaggagcagcatcttcaatgacgtgaaa ggccttctttgcttgtatgaagcatgttttctagcagtgcatggagaagatatattagaagaagcactttgttttacaaagaaacatctcaaaacttta gctgaaaattcaagccccatcttcagaaacttataaagaatgctttggtttttccaagtcaccacaacatggaaaggettgatgctttgcattatat atccttctacgaagcagatgaatctccaaacgaaactcttctcagatttgcaaagttggattttaatcgcctacaattactttacaagaaagagttg gctttagttacggcgtggtggaaagatacaaatcttgtggaaagtgttccgtatggaagaaatagagtttcagagtcatacatatgggcacttgg aaacctttcgagcctcagtacagttttctcgaatggetttatgtaagtatgtacaattggaaacggtgctagatgacacatatgatacatatggta ccgttgatgaactgacaacttttacagctgcgctagaaaggttcactaccgatggtgtcgatgagctaccagagtatataaaatctttctacatgta tatattgaggttttatgaagaagcggagaatgatgtcactgaaagatgcttttataaagtttctttgctagagaaatgttcaaagagttggcgaaa ggctattttcaggagaaaatatggaacaatgatggggaaacgccgtccttcgatgagtacatgaggaatggaaaagtgagcagcacttttgac tgttgtgcagcgccagttatccaggaaatggaaaacgcgggtataaaggaaatgatatgggtacgaagtgatccaccgattattgcttgtgcca agttcatcggtcgtctcatgaatgatgtaccaggaaaagtggatgagacaaagagagaagactacccaaaagtatggattgctatatgaaac aatatggggtgtcacgaggtgaggccattgaagccatcctaaaaatagttgaaaatagttggaaggacatgaatgaagattttatgaagacaac aaatgtgcctaaaataatgctaaagtacactttcaactatgctcgtatggcaacggttttctacacgggaactgatatgtttacatatgaatcatcct caaaacagttaatcacttccatgttcctcaaaccacttcctatttaa

Recombinant EfCAS (blue: encoded by the sequence deriving from pCold TF vector; black: EfCAS)

MNHHHHHHIEGRHMMAKVEHDVLRGASKNVPNGIWGYSLASISPSLDLELE SHGEEVEAIKEKVKDMLIESAKELTCNIEFINLLCRLGVSYYFEVEIDNQLDHIF NNILPDIVEDNDYDLSTLATLFRVLRQHGHKMTSDVFKKFKSRDGEFRSSIFN DVKGLLCLYEACFLAVHGEDILEEALCFTKKHLKTLAENSSPHLQKLIKNALV FPSHHNMERLDALHYISFYEADESPNETLLRFAKLDFNRLQLLYKKELALVTA WWKDTNLVESVPYGRNRVSESYIWALGNLFEPQYSFSRMALCKYVQLETVL DDTYDTYGTVDELTTFTAALERFTTDGVDELPEYIKSFYMYILRFYEEAENDV TERCFYKVSFAREMFKELAKGYFQEKIWNNDGETPSFDEYMRNGKVSSTFDC CAAPVIQEMENAGIKEMIWVRSDPPIIACAKFIGRLMNDVPGKVDETKREDYP KSMDCYMKQYGVSRGEAIEAILKIVENSWKDMNEDFMKTTNVPKIMLKYTF NYARMATVFYTGTDMFTYESSSKQLITSMFLKPLPI 